\title{
Randomly driven granular fluids: Collisional statistics and short scale structure
}

\author{
I. Pagonabarraga \\ Departament de Física Fonamental, Universitat de Barcelona, Av. Diagonal 647, 08028-Barcelona, Spain \\ E. Trizac \\ Laboratoire de Physique Théorique, * Bâtiment 210, Université Paris-Sud, 91405 Orsay Cedex, France \\ T. P. C. van Noije and M. H. Ernst \\ Instituut voor Theoretische Fysica, Universiteit Utrecht, Postbus 80006, 3508 TA Utrecht, The Netherlands
}

(Received 26 July 2001; published 14 December 2001)

\begin{abstract}
We present a molecular-dynamics and kinetic theory study of granular material, modeled by inelastic hard disks, fluidized by a random driving force. The focus is on collisional averages and short-distance correlations in the nonequilibrium steady state, in order to analyze in a quantitative manner the breakdown of molecular chaos, i.e., factorization of the two-particle distribution function, $f^{(2)}\left(x_{1}, x_{2}\right) \simeq \chi f^{(1)}\left(x_{1}\right) f^{(1)}\left(x_{2}\right)$ in a product of single-particle ones, where $x_{i}=\left\{\mathbf{r}_{i}, \mathbf{v}_{i}\right\}$ with $i=1,2$ and $\chi$ represents the position correlation. We have found that molecular chaos is only violated in a small region of the two-particle phase space $\left\{x_{1}, x_{2}\right\}$, where there is a predominance of grazing collisions. The size of this singular region grows with increasing inelasticity. The existence of particle- and noise-induced recollisions magnifies the departure from mean-field behavior. The implications of this breakdown in several physical quantities are explored.
\end{abstract}

DOI: 10.1103/PhysRevE.65.011303

PACS number(s): 45.70. $-\mathrm{n}, 51.10 .+\mathrm{y}$

\section{INTRODUCTION}

The interesting phenomena observed in recent experiments with mono- and multi-layers of granular material on vibrating plates $[1-4]$ show the need to develop kinetic theories for rapid granular flows with mechanisms for energy input, different from those in shear flows or flows through vertical pipes. In the present paper, the fluidization is driven by a random external force, which gives frequent kicks to each particle in between collisions. Such a driving mechanism has recently been studied by many authors [ $5-11]$. The basic physical interest is the understanding of the nonequilibrium stationary states (NESS) that exist in the presence of this random force. The advantage of this fluidization mechanism, besides its potential physical realizations, lies in the fact that the NESS is linearly stable against spatial inhomogeneities.

In Ref. [10], to which we will refer to as paper I, we have studied the large-scale structure and presented a hydrodynamic description of randomly driven granular fluids, modeled as systems of smooth inelastic hard spheres (IHS). The IHS model accounts for two essential features of granular matter: hard-core exclusion and dissipative collisions [12]. The dynamics is described by a constant coefficient $\alpha$ of normal restitution. In collisions, a fraction of the relative kinetic energy is lost, which is proportional to the inelasticity $\epsilon=1-\alpha^{2}$. The stochastic external force compensates this energy loss, and drives the IHS fluid into a NESS. This stationary state, though homogeneous and stable against spatial fluctuations on large space and time scales (at least for weakly inelastic spheres), was shown to exhibit long-range spatial correlations in density, velocity, and granular tem-

\footnotetext{
*Unité Mixte de Recherche UMR 8627 du CNRS.
}

perature fields that extend much beyond the mean free path. In fact, the corresponding structure functions $S(k)$ diverge as $1 / k^{2}$ as the wave-number $k \rightarrow 0$, a behavior caused by the random external force, which does not conserve momentum whereas the collisions between particles do. These longrange correlations are of algebraic form, $\sim 1 / r^{d-2}$, which corresponds to $\ln r$ in two dimensions $(d=2)$. The existence of such extremely long-range spatial correlations is one example of the many nontrivial properties of nonequilibrium stationary states in general $[13,14]$.

Differences in the stationary states between fluids with dissipative and conservative interactions also manifest themselves in the kinetic properties of the fluid, such as the velocity distribution function, which deviates from a Maxwellian, in particular, in the high-energy tail of the distribution. In Ref. [9], the existence of an overpopulated high-energy tail $\hat{f} \sim \exp \left[-C v^{3 / 2}\right]$, where $C$ is a constant that depends on the inelasticity, has been obtained from kinetic theory. A similar behavior has been observed experimentally at highvibrational accelerations $[3,4]$. This observation indicates that certain features of the experiment might be reproduced by modeling the input of energy into the horizontal motion of the beads by a random external force, although other energy injection mechanisms that could be relevant to recover the large-velocity tail have been put forward [15]. In similar experiments [2], with a vertically vibrating plate covered with a monolayer of steel balls with a packing fraction around 50\%, the velocity distribution of the horizontal velocities has been measured, and again, overpopulated non-Gaussian highenergy tails have been observed. In the present paper, we will investigate the kinetic properties and short-scale correlations that characterize the stationary state. More specifically, we will compare molecular-dynamics (MD) simulations of inelastically colliding disks with analytic predictions based on the assumption of molecular chaos. 
The Boltzmann equation for dilute gases of particles that interact via short-ranged repulsive interactions is based on the assumption of molecular chaos, also called the Stosszahlansatz or mean-field approximation. It assumes that the velocities of colliding particles just before collisions are uncorrelated, i.e., their pair-distribution function factorizes, $\hat{f}^{(2)}\left(x_{1}, x_{2}, t\right)=\hat{f}\left(x_{1}, t\right) \hat{f}\left(x_{2}, t\right)$, where $x_{i}=\left\{\mathbf{r}_{i}, \mathbf{v}_{i}\right\}$ denotes the position and velocity of particle $i$. Enskog's extension of the Boltzmann equation to a dense system of hard spheres [16], referred to as Enskog-Boltzmann equation, is also based on the fundamental assumption of the absence of velocity correlations. Here, the assumption of molecular chaos postulates that $\hat{f}^{(2)}\left(x_{1}, x_{2}, t\right)=\chi \hat{f}\left(x_{1}, t\right) \hat{f}\left(x_{2}, t\right)$ for approaching particles $\left(\mathbf{v}_{12} \cdot \mathbf{r}_{12}<0\right)$ just before collision $\left(r_{12}\right.$ $=\sigma+0)$, where $\chi$ is assumed to be the radial pairdistribution function at contact $g\left(r_{12}=\sigma+0\right)$ in local equilibrium. It implies the additional assumption that spatial correlations between colliding particles just before collision are independent of their velocities, i.e., the absence of positionvelocity correlations. The Enskog $\chi$ factor enhances the collision frequency at higher densities. For dilute gases, the assumption of molecular chaos seems to be justified. Recently, Lutsko [17] and Soto and Mare-schal [18] derived for a freely evolving inelastic hard disk fluid a relation between pre- and post-collision radial distribution function at contact, as a function of the angle, $\theta=\cos ^{-1}\left(\hat{\mathbf{v}}_{12} \cdot \hat{\mathbf{r}}_{12}\right)$, between the relative velocity $\mathbf{v}_{12}$ of the colliding particles, and their relative position at contact $\mathbf{r}_{12}$, and they confirmed their results by MD simulations. Their observations made it clear that further arguments are needed to clarify the meaning of the $\chi$ factor in Enskog's formulation of the molecular chaos assumption. This will be done in Sec. II A.

The breakdown of molecular chaos at higher densities in classical fluids with conservative forces has been extensively investigated in the 1960s and 1970s [19]. This breakdown is caused by sequences of correlated binary collisions, the socalled ring collisions [20]. They lead to long-time tails in velocity and stress autocorrelation functions [21,22], and to long-range spatial correlations in NESS [13]. The quantitative effects of velocity correlations on transport coefficients at liquid densities are also significant. For instance, molecular-dynamics simulations on elastic hard-sphere systems at liquid densities [23] have shown that the long- time tails increase the measured self-diffusion coefficient $D$ typically by 15 to $20 \%$ with respect to the prediction of the Enskog theory $D_{\mathrm{E}}=D_{\mathrm{B}} / \chi$, where $D_{\mathrm{B}}$ is the Boltzmann value of the self-diffusion coefficient.

A well-known example of short-scale structure in granular fluids are the position-velocity correlations leading to the phenomenon of inelastic collapse [24,25], which is a divergence of the collision frequency $\omega$ in a finite time. The collapse singularity implies that an infinite number of collisions occurs within a finite-time interval in a subset of (nearly) touching particles, ordered in linear arrays. The phenomenon is, however, an artifact of the assumption that the coefficient of restitution $\alpha$ is independent of the impact velocities, whereas on physical grounds $\alpha\left(v_{12}\right) \rightarrow 1$ (elastic limit), as the relative velocity $v_{12}$ vanishes. Molecular-dynamics simu- lations have shown that the assumption of molecular chaos is also violated in undriven granular fluids in their late stages of evolution, the so-called nonlinear clustering regime. For instance, the measured distribution of impact parameters is not uniform, as expected on the basis of molecular chaos, but biased toward grazing collisions [26-28].

As shown below, in the driven IHS fluid there is an important additional reason for the breakdown of molecular chaos, namely, the strong increase in collision frequency at small relative velocities between two isolated particles, caused by the so-called noise- induced re-collisions. This correction to the collision frequency, that is important at all densities, is also neglected in the molecular chaos assumption.

The main goal of this paper is to quantify, analyze, and interpret the effects of the breakdown of molecular chaos in the NESS of inelastic hard spheres that are subject to a random external force between collisions. We will focus in particular on velocity-velocity correlations and position-velocity correlations between particles almost in contact, i.e., the short-scale structure.

Section II presents the analytic results, based on the Enskog-Boltzmann equation, which has been modified to account for the external energy input. In Sec. III, we present molecular-dynamics results for several quantities that characterize the collision processes and related short-scale structure of the NESS, and make a comparison with predictions based on molecular chaos.

\section{KINETIC THEORY FOR THE NESS}

\section{A. Molecular chaos and Enskog approximation}

The Enskog-Boltzmann equation for the single-particle distribution $\hat{f}\left(\mathbf{v}_{1}, t\right)$ in a spatially homogeneous randomly driven fluid of inelastic hard spheres of diameter $\sigma$ reads in $d=2$ or 3 dimensions [9]

$$
\begin{aligned}
\partial_{t} \hat{f}\left(\mathbf{v}_{1}, t\right)= & n \chi \sigma^{d-1} \int \mathrm{d} \mathbf{v}_{2} \int \mathrm{d} \hat{\boldsymbol{\sigma}} \Theta\left(\mathbf{v}_{12} \cdot \hat{\boldsymbol{\sigma}}\right)\left(\mathbf{v}_{12} \cdot \hat{\boldsymbol{\sigma}}\right) \\
& \times\left\{\frac{1}{\alpha^{2}} \hat{f}\left(\mathbf{v}_{1}^{* *}, t\right) \hat{f}\left(\mathbf{v}_{2}^{* *}, t\right)-\hat{f}\left(\mathbf{v}_{1}, t\right) \hat{f}\left(\mathbf{v}_{2}, t\right)\right\} \\
& +\frac{\xi_{0}^{2}}{2}\left(\frac{\partial}{\partial \mathbf{v}_{1}}\right)^{2} \hat{f}\left(\mathbf{v}_{1}, t\right),
\end{aligned}
$$

where $\mathbf{v}_{12}=\mathbf{v}_{1}-\mathbf{v}_{2}$ and $n$ the number density. The Heaviside function $\Theta(x)$ restricts the $\hat{\boldsymbol{\sigma}}$ integration to the hemisphere $\mathbf{v}_{12} \cdot \hat{\boldsymbol{\sigma}}>0$, where $\hat{\boldsymbol{\sigma}}$ is the unit vector along the line of centers of the colliding spheres at contact, pointing from particle 2 to 1 . In the sequel $\hat{\mathbf{a}}=\mathbf{a} /|\mathbf{a}|$ denotes a unit vector. The gain term of the collision integral describes the restituting collisions that convert the precollision velocities $\left(\mathbf{v}_{1}^{* *}, \mathbf{v}_{2}^{* *}\right)$ into $\left(\mathbf{v}_{1}, \mathbf{v}_{2}\right)$, while the loss term describes the direct collisions, and contains the precollision velocities $\left(\mathbf{v}_{1}, \mathbf{v}_{2}\right)$ leading to postcollision velocities $\left(\mathbf{v}_{1}^{*}, \mathbf{v}_{2}^{*}\right)$. The postcollision and restituting velocities have been defined in [29]. The $\chi$ factor will be discussed below. 
As derived in [9], the Fokker-Planck term accounts for the external energy input, and describes diffusion in velocity space with a diffusivity proportional to the rate of energy input $\xi_{0}^{2}$ per unit mass. Here, $\xi_{0}$ is the strength of the random driving force, which is assumed to be Gaussian white noise $[9,10]$.

Before studying the short-scale structure, we consider the single- particle distribution function $\hat{f}(\mathbf{v})$ in the NESS. The stationary solution of Eq. (1) is determined by the balance $m \xi_{0}^{2}=\Gamma$, of external heating $m \xi_{0}^{2}$, and internal loss of energy due to collisions, $\Gamma$. It is characterized by a time-independent temperature $T=\left\langle m v^{2} / d\right\rangle$ defined as the average kinetic energy per particle, and discussed in paper I. As mentioned in the introduction, this stationary solution exhibits an overpopulated high-energy tail $\hat{f} \sim \exp \left[-C v^{3 / 2}\right]$. The structure of the tail distribution is determined by collisions of very energetic particles with "thermal" particles, and may be obtained by neglecting the gain term in the Boltzmann equation [9].

In Ref. [9], $\hat{f}(\mathbf{v})$ has been calculated by solving the Enskog-Boltzmann Eq. (1) by an expansion in Sonine polynomials. To formulate this result, it is convenient to introduce a rescaled distribution function $f(\mathbf{c})$, defined by $\hat{f}(\mathbf{v})$ $\equiv\left[1 / v_{0}^{d}\right] f(\mathbf{c})$ with $\mathbf{c} \equiv \mathbf{v} / v_{0}$, where $v_{0} \equiv \sqrt{2 T / m}$ is the thermal velocity and $d$ the dimensionality. This gives

$f(\mathbf{c})=\varphi(c)\left\{1+a_{2}\left[\frac{1}{2} c^{4}-\frac{1}{2}(d+2) c^{2}+\frac{1}{8} d(d+2)\right]+\cdots\right\}$,

where the Maxwellian $\varphi(c) \equiv \pi^{-d / 2} \exp \left(-c^{2}\right)$. Note that $a_{2}$ is proportional to the fourth cumulant of the scaling form $f(\mathbf{c})$, i.e.,

$$
a_{2}=\frac{4}{d(d+2)}\left[\left\langle c^{4}\right\rangle-\frac{1}{4} d(d+2)\right]=\frac{4}{3}\left[\left\langle c_{x}^{4}\right\rangle-3\left\langle c_{x}^{2}\right\rangle^{2}\right],
$$

and vanishes in the elastic limit. An explicit calculation to linear order in $a_{2}$ gives [9]

$$
a_{2}=\frac{16(1-\alpha)\left(1-2 \alpha^{2}\right)}{73+56 d-24 \alpha d-105 \alpha+30(1-\alpha) \alpha^{2}} .
$$

In the next section, this prediction will be tested against molecular-dynamics simulations.

Consider first the exact expression for the mean collision frequency in the homogeneous NESS, defined as

$$
\begin{aligned}
\omega= & n \sigma^{d-1} \int \mathrm{d} \mathbf{v}_{1} \int \mathrm{d} \mathbf{v}_{2} \int \mathrm{d} \hat{\boldsymbol{\sigma}} \Theta \\
& \times\left(-\mathbf{v}_{12} \cdot \hat{\boldsymbol{\sigma}}\right)\left|\mathbf{v}_{12} \cdot \hat{\boldsymbol{\sigma}}\right| \hat{f}^{(2)}\left(\mathbf{v}_{1}, \mathbf{v}_{2}, \boldsymbol{\sigma}\right),
\end{aligned}
$$

where $\hat{f}^{(2)}\left(\mathbf{v}_{1}, \mathbf{v}_{2}, \boldsymbol{\sigma}\right)$ is the dynamic or constrained pairdistribution function with velocities aiming to collide, just before contact with $\mathbf{r}_{12}=\boldsymbol{\sigma}$. Molecular chaos, also referred to as mean-field theory, requires the complete factorization of the dynamic precollisional pair function,

$$
\hat{f}^{(2)}\left(\mathbf{v}_{1}, \mathbf{v}_{2}, \boldsymbol{\sigma}\right)=\chi \hat{f}\left(\mathbf{v}_{1}\right) \hat{f}\left(\mathbf{v}_{2}\right) .
$$

What is the meaning of the $\chi$ factor, used in formulating the molecular chaos hypothesis? This hypothesis for dilute gases concerns the absence of correlations in precollision velocities, and in precollision positions $(\chi=1)$. In dense fluids on the other hand, the precollision position correlation $\chi$ is different from 1 , but the precollision velocity-velocity and position-velocity correlations are still assumed to be absent.

In the literature, it is common to take $\chi$ equal to the radial distribution function at contact in local equilibrium, i.e., $\chi$ $=\chi_{\mathrm{E}} \equiv g_{\text {eq }}(r \rightarrow \sigma+0)$, which mainly accounts for precollision hard-core exclusion effects. For hard disks and hard spheres, the latter function is approximately given by the Verlet-Levesque (2D) and Carnahan-Starling (3D) approximations [31],

$$
\begin{gathered}
\chi_{\mathrm{E}}(\phi)=\left(1-\frac{7}{16} \phi\right) /(1-\phi)^{2} \quad(2 \mathrm{D}), \\
\chi_{\mathrm{E}}(\phi)=(2-\phi) / 2(1-\phi)^{3} \quad(3 \mathrm{D}),
\end{gathered}
$$

where the packing fraction in $d$ dimensions is defined as $\phi$ $=n(\sigma / 2)^{d} \Omega_{d} / d$, and $\Omega_{d}=2 \pi^{d / 2} / \Gamma(d / 2)$ is the surface area of a $d$-dimensional unit sphere. In this paper, we refer to the molecular chaos approximation with $\chi=\chi_{\mathrm{E}}$, as the Enskog approximation.

In principle, different options are open for the $\chi$ factor. As $\hat{f}^{(2)}$ is the dynamic precollision pair-distribution function at contact, an alternative choice for the $\chi$ in the factorized form could be the dynamic precollision radial distribution function at contact, defined as an average over the precollision hemisphere,

$\chi^{(-)} \equiv\left[2 / \Omega_{d}\right] \int d \mathbf{v}_{1} \int d \mathbf{v}_{2} \int \mathrm{d} \hat{\boldsymbol{\sigma}} \Theta\left(-\mathbf{v}_{12} \cdot \hat{\boldsymbol{\sigma}}\right) \hat{f}^{(2)}\left(\mathbf{v}_{1}, \mathbf{v}_{2}, \boldsymbol{\sigma}\right)$.

Another option could be the unconstrained radial distribution, $g(r)$, in the NESS, extrapolated to contact $(r \rightarrow \sigma)$. This function is further discussed in Sec. II D.

For the randomly heated fluid under study here, the dynamics is not purely hard-sphere like. The random force acting on the particles may be expected to contribute to the value of the pair- distribution function at contact. This effect will be addressed in the subsequent sections.

Equation (5) with $\hat{f}(\mathbf{v})$ replaced by the Maxwellian, yields for the collision frequency in the molecular chaos approximation $\omega_{\mathrm{mc}}(T)=\chi \omega_{0}(T)$, and more specifically, in the Enskog approximation,

$$
\omega_{\mathrm{E}}(T)=\chi_{\mathrm{E}} \omega_{0}(T) .
$$

Here, the Boltzmann collision frequency for dilute gases is given by

$$
\omega_{0}(T)=\Omega_{d} n \sigma^{d-1} \sqrt{T / \pi m},
$$

and the small correction of $\mathcal{O}\left(a_{2}\right)$ appearing in Eq. (2) has been neglected. 
Spatial correlation functions in nonequilibrium stationary states are quite different from local equilibrium ones, and show long-range correlations due to correlated sequences of ring collisions, also referred to as mode-coupling effects [13]. In paper I, we have shown the existence of very longrange correlations $\sim 1 / r^{d-2}$ in the randomly driven IHS fluid. The short-range correlations in the NESS may, in principle, be obtained from the ring kinetic equation for IHS (see Ref. [29]). However, systematic methods to evaluate collision integrals and pair-correlation functions at short distances using this ring kinetic theory have not yet been developed. In the section on simulation results, we return to the effects of ring collisions, and present arguments why their contributions are expected to be more important here than for elastic hard spheres.

\section{B. Collisional averages}

In hard-sphere systems, there are many properties that involve the pair-distribution function of particles just before collision. To study these, it is convenient to introduce the collisional average $\langle\ldots\rangle_{\text {coll }}$ for a quantity $A$ in the NESS, defined as

$$
\left\langle A\left(\mathbf{v}_{1}, \mathbf{v}_{2}, \boldsymbol{\sigma}\right)\right\rangle_{\mathrm{coll}}=\frac{\int \mathrm{d} \mathbf{v}_{1} \int \mathrm{d} \mathbf{v}_{2} \int \mathrm{d} \hat{\boldsymbol{\sigma} \Theta}\left(-\mathbf{v}_{12} \cdot \hat{\boldsymbol{\sigma}}\right)\left|\mathbf{v}_{12} \cdot \hat{\boldsymbol{\sigma}}\right| A\left(\mathbf{v}_{1}, \mathbf{v}_{2}, \boldsymbol{\sigma}\right) \hat{f}^{(2)}\left(\mathbf{v}_{1}, \mathbf{v}_{2}, \boldsymbol{\sigma}\right)}{\int \mathrm{d} \mathbf{v}_{1} \int \mathrm{d} \mathbf{v}_{2} \int \mathrm{d} \hat{\boldsymbol{\sigma} \Theta}\left(-\mathbf{v}_{12} \cdot \hat{\boldsymbol{\sigma}}\right)\left|\mathbf{v}_{12} \cdot \hat{\boldsymbol{\sigma}}\right| \hat{f}^{(2)}\left(\mathbf{v}_{1}, \mathbf{v}_{2}, \boldsymbol{\sigma}\right)} .
$$

In the sequel, it is more convenient to work with a rescaled pair-distribution function $\hat{f}^{(2)}\left(\mathbf{v}_{1}, \mathbf{v}_{2}, \mathbf{r}_{12}\right)$ $=\left[1 / v_{0}^{2 d}\right] f^{(2)}\left(\mathbf{c}_{1}, \mathbf{c}_{2}, \mathbf{r}_{12}\right)$. To express the collisional averages (11) in rescaled variables, one replaces $\hat{f}^{(2)}$ by $f^{(2)}, \mathbf{v}_{i}$ by $\mathbf{c}_{i}, \mathbf{v}_{12}$ by $\mathbf{g}=\mathbf{c}_{1}-\mathbf{c}_{2}$, and $A\left(\mathbf{v}_{1}, \mathbf{v}_{2}, \boldsymbol{\sigma}\right)$ by $A\left(v_{0} \mathbf{c}_{1}, v_{0} \mathbf{c}_{2}, \boldsymbol{\sigma}\right)$.

These objects may be conveniently computed in eventdriven molecular-dynamics algorithms for hard-sphere systems [30]. Collisional averages are defined for particles that are about to collide (i.e., $\left|r_{12}\right|=\sigma+0$ ), and may be calculated from kinetic theory using the molecular chaos assumption, possibly supplemented with the Enskog approximation at higher densities.

Collisional averages of great importance are the collisional energy loss per unit time $(d / 2) n \Gamma$, and the excess hydrostatic pressure $p-n T$, resulting from collisional transfer of momentum. With a minor generalization to $d$ dimensions, we obtain from Ref. [32] the exact expression for the pressure in the NESS

$$
\begin{aligned}
\frac{p(T)}{n T}-1= & \left(\frac{1+\alpha}{2 d}\right) n \sigma^{d} \int \mathrm{d}_{1} \int \mathrm{d} \mathbf{c}_{2} \int \mathrm{d} \hat{\boldsymbol{\sigma}} \\
& \times \Theta(-\mathbf{g} \cdot \hat{\boldsymbol{\sigma}})|\mathbf{g} \cdot \hat{\boldsymbol{\sigma}}|^{2} f^{(2)}\left(\mathbf{c}_{1}, \mathbf{c}_{2}, \boldsymbol{\sigma}\right) \\
= & \left(\frac{1+\alpha}{2 d}\right) \frac{\sigma \omega}{v_{0}}\langle|\mathbf{g} \cdot \hat{\boldsymbol{\sigma}}|\rangle_{\mathrm{coll}} .
\end{aligned}
$$

The second equality is obtained by introducing the collisional average (11) and expressing its denominator in terms of the collision frequency given by Eq. (5). In fact, inserting Eq. (6) into the first line of Eq. (12) allows one to carry out the $\hat{\sigma}$ integration, and the right-hand side becomes proportional to the rescaled velocity average $\int \mathrm{d} \mathbf{c}_{1} \int \mathrm{d} \mathbf{c}_{2} g^{2} f\left(\mathbf{c}_{1}\right) f\left(\mathbf{c}_{2}\right)=2$ without any further assumption about neglecting the term proportional to $a_{2}$ in Eq. (2). This argument is special for the pressure, as other moments involve the knowledge of the complete $f(c)$. Indeed, the generic collisional average becomes $\left\langle|\mathbf{g} \cdot \hat{\boldsymbol{\sigma}}|^{m}\right\rangle_{\text {coll }}$ $=2^{m / 2} \Gamma[(1 / 2) m+1]$, independent of dimensionality, assuming molecular chaos (6) and replacing $f(c)$ by the Maxwellian $\varphi(c)$ (the contributions coming from $a_{2}$ are quite small and may be neglected; they have been computed in [9]). Finally, the pressure-may be expressed as

$$
\frac{p_{\mathrm{mc}}(T)}{n T}-1=2^{d-2}(1+\alpha) \chi \phi .
$$

Different choices for $\chi$ yield different approximations. For instance, with $\chi=\chi_{\mathrm{E}}$, we obtain the Enskog approximation $p_{\mathrm{E}}(T)$ for the pressure of IHS.

In the elastic limit $p_{\mathrm{E}}(T)$ at $\alpha=1$ gives the standard equation of state for elastic hard disks or spheres. Notice that the pressure for IHS is only defined kinetically as the momentum flux, which leads to Eq. (12). A statistical mechanical derivation of the equation of state from the partition function or free energy for the IHS fluid does not exist.

In a similar manner, we obtain the exact expression for the collisional damping rate

$$
\begin{aligned}
\Gamma(T)= & \left(\frac{1-\alpha^{2}}{2 d}\right) n \sigma^{d-1} v_{0} T \int \mathrm{d} \mathbf{c}_{1} \int \mathrm{d} \mathbf{c}_{2} \int \mathrm{d} \hat{\boldsymbol{\sigma}} \\
& \times \Theta(-\mathbf{g} \cdot \hat{\boldsymbol{\sigma}})|\mathbf{g} \cdot \hat{\boldsymbol{\sigma}}|^{3} f^{(2)}\left(\mathbf{c}_{1}, \mathbf{c}_{2}, \boldsymbol{\sigma}\right) \\
= & \gamma_{0} \omega T\left\langle|\mathbf{g} \cdot \hat{\boldsymbol{\sigma}}|^{2}\right\rangle_{\mathrm{coll}}=m \xi_{0}^{2},
\end{aligned}
$$

where $\gamma_{0}=\left(1-\alpha^{2}\right) / 2 d$ is the dimensionless damping constant introduced in Refs. $[9,10]$. The last equality (14) expresses the balance between the energy input due to the white noise, and the collisional loss of energy in the NESS, and determines the temperature $T$ in the NESS. By special- 

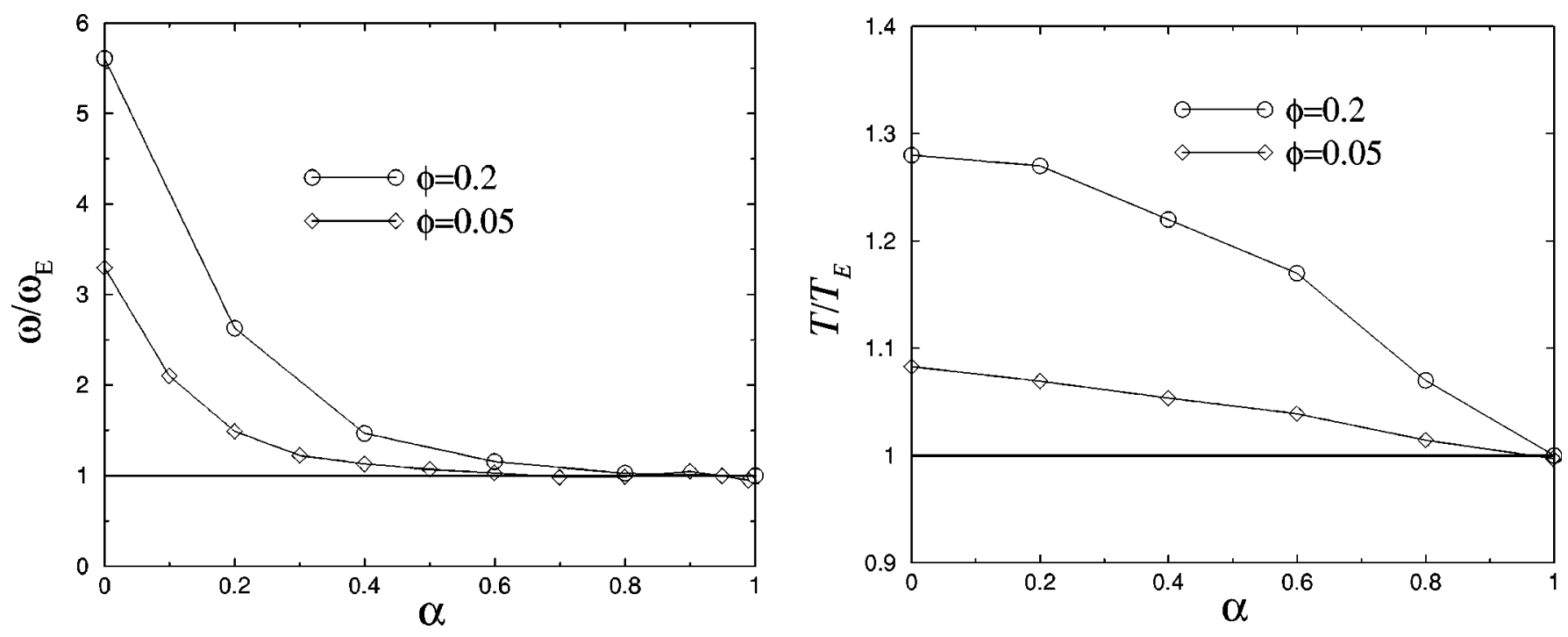

FIG. 1. Kinetic temperature $T / T_{\mathrm{E}}$ and collision frequency $\omega / \omega_{\mathrm{E}}$ where $T_{\mathrm{E}}$ and $\omega_{\mathrm{E}}$ are defined in Eqs. (17) and (19), for a packing fractions $\phi=0.05$ and $\phi=0.2$.

izing this equation to the Enskog mean-field approximation, $f^{(2)}=\chi_{\mathrm{E}} f f$, where $\left\langle|\mathbf{g} \cdot \hat{\boldsymbol{\sigma}}|^{2}\right\rangle_{\mathrm{coll}}=2$, we obtain the approximate result

$$
\Gamma_{\mathrm{E}}(T)=2 \gamma_{0} \omega_{\mathrm{E}}(T) T
$$

and similar relations for different choices of $\chi$. It is convenient to define a reference temperature $T_{\mathrm{E}}$ through the relation

$$
\Gamma_{\mathrm{E}}\left(T_{\mathrm{E}}\right)=m \xi_{0}^{2},
$$

or more explicitly

$$
T_{\mathrm{E}}=m\left(\frac{\xi_{0}^{2} \sqrt{\pi}}{2 \gamma_{0} \Omega_{d} \chi_{\mathrm{E}} n \sigma^{d-1}}\right)^{2 / 3} .
$$

Moreover, the definition of $T_{\mathrm{E}}$ combined with the NESS condition $\Gamma(T)=m \xi_{0}^{2}$ implies the relation $\Gamma(T)=\Gamma_{\mathrm{E}}\left(T_{\mathrm{E}}\right)$, and consequently,

$$
\frac{\Gamma(T)}{\Gamma_{\mathrm{E}}(T)}=\frac{\Gamma_{\mathrm{E}}\left(T_{\mathrm{E}}\right)}{\Gamma_{\mathrm{E}}(T)}=\left(\frac{T_{\mathrm{E}}}{T}\right)^{3 / 2} .
$$

In the sequel, we will also use a reference frequency $\omega_{\mathrm{E}}$ without any argument to denote

$$
\omega_{\mathrm{E}} \equiv \omega_{\mathrm{E}}\left(T_{\mathrm{E}}\right)=\chi_{\mathrm{E}} \Omega_{d} n \sigma^{d-1} \sqrt{T_{\mathrm{E}} / \pi m} .
$$

Although we will discuss the simulation results in detail in the next section, it is of interest already at this point to note that for these systems, as shown in Fig. 1, the ratio of the kinetic temperature and the reference temperature, $T / T_{\mathrm{E}}$, is only somewhat larger than one for all $\alpha$, that it approaches one in the elastic limit $(\alpha \rightarrow 1)$, and that it monotonically increases with decreasing $\alpha$ (see Fig. 1). The same figure shows that the ratio, $\omega / \omega_{\mathrm{E}}$ also approaches one for $\alpha \rightarrow 1$, with a steep increase to a value 5.6 as $\alpha \rightarrow 0$. Further discus- sion of these points is postponed untill Sec. III. If the Enskog factorization $\hat{f}^{(2)}=\chi_{\mathrm{E}} \hat{f} \hat{f}$ would be exact, then $T=T_{\mathrm{E}}$ and $\omega$ $=\omega_{\mathrm{E}}$.

A third quantity of interest, the precollisional $\chi^{(-)}$factor, defined in Eq. (8), may also be expressed as a collisional average using Eq. (11),

$$
\chi^{(-)}=\left(2 \omega / \Omega_{d} n \sigma^{d-1} v_{0}\right)\left\langle|\mathbf{g} \cdot \hat{\boldsymbol{\sigma}}|^{-1}\right\rangle_{\mathrm{coll}} .
$$

Before closing this section, a caveat about internal consistency is appropriate. To obtain consistent theoretical predictions for the pressure $p$ or dissipation rate $\Gamma$, it is paramount that both factors $\omega$ and $\left\langle|\mathbf{g} \cdot \boldsymbol{\sigma}|^{m}\right\rangle_{\text {coll }}$ be calculated using identical approximations for $f^{(2)}$. For instance, the mean-field or molecular chaos approximation for the dissipation rate, $\Gamma_{\mathrm{E}}(T)=2 \gamma_{0} \omega T$, —an expression commonly used in granular hydrodynamic equations-should necessarily be combined with $\omega_{\mathrm{E}}(T)$ in Eq. (9). Any improved theoretical calculation for $\omega$ without a concomitant correction to the mean-field result for $\left\langle|\mathbf{g} \cdot \boldsymbol{\sigma}|^{m}\right\rangle_{\text {coll }}$ is inconsistent.

\section{Velocity distributions}

We study a variety of collisional averages and corresponding probability distributions. By choosing $A\left(\mathbf{c}_{1}, \mathbf{c}_{2}, \boldsymbol{\sigma}\right)$ $=\delta(|\mathbf{g}|-g)$ we obtain the probability $P_{r}(g)$ that two colliding particles have a relative speed $\left|\mathbf{c}_{12}\right|=g$. From here on, we only quote results for two dimensions. Analytic calculations are based on the molecular chaos assumption (6) in combination with Eq. (2). Inspection of Eq. (11) shows that under this assumption, the collisional averages are independent of the $\chi$ factor. Straightforward algebra gives for the constrained $g$ distribution,

$$
\begin{aligned}
P_{r}(g) & =\left\langle\delta\left(\left|\mathbf{c}_{12}\right|-g\right)\right\rangle_{\text {coll }} \\
& =\sqrt{\frac{2}{\pi}} g^{2} e^{-1 / 2 g^{2}}\left\{1+\frac{1}{16} a_{2}\left(g^{4}-8 g^{2}+9\right)\right\} .
\end{aligned}
$$


Similarly, we obtain the probability distribution for the center of mass velocity $\mathbf{G} \equiv 1 / 2\left(\mathbf{c}_{1}+\mathbf{c}_{2}\right)$,

$$
P_{\mathrm{CM}}(G)=\langle\delta(|\mathbf{C}|-G)\rangle_{\mathrm{coll}}=4 G e^{-2 G^{2}}\left\{1+a_{2}\left(G^{4}-G^{2}\right)\right\} .
$$

It equals the unconstrained equilibrium distribution function apart from a small term of $\mathcal{O}\left(a_{2}\right)$. Furthermore, the probability that the precollision speed $\left|\mathbf{c}_{i}\right|$ of one of the colliding particles $(i=1,2)$ has a value $v$ is

$$
\begin{aligned}
P(v) & =\left\langle\delta\left(\left|\mathbf{c}_{1}\right|-v\right)\right\rangle_{\text {coll }} \\
& =\sqrt{2} v e^{-3 v^{2} / 2}\left\{\left(1+v^{2}\right) I_{0}\left(\frac{1}{2} v^{2}\right)+v^{2} I_{1}\left(\frac{1}{2} v^{2}\right)\right\},
\end{aligned}
$$

whereas the unconstrained distribution is $\sim v \exp \left(-v^{2}\right)$. In evaluating this collisional average, we neglect the $a_{2}$ contribution, and carry out the constrained $\hat{\boldsymbol{\sigma}}$ integration. To calculate the remaining integral $\int \mathrm{d}_{2} c_{12} \varphi\left(c_{2}\right)$, we change integration variables to $\mathbf{g}$ expressed in polar coordinates $\{g, \phi\}$, and use the relation $\int_{0}^{\pi} \mathrm{d} \phi \exp \left(-2 c_{1} g \cos \phi\right)=\pi I_{0}\left(2 c_{1} g\right)$. The subsequent $g$ integration follows from (6.618.4) in Ref. [33]. Using the asymptotic expressions $I_{0}(x) \sim I_{1}(x)$ $\sim \exp (x) / \sqrt{2 \pi x}$ for the modified Bessel functions of the zeroth and first order, we obtain the high-energy behavior $P(v) \sim 2 \sqrt{2 / \pi} v^{2} \exp \left(-v^{2}\right)$.

In a similar manner, we obtain the following velocity moments and correlations, using the molecular chaos assumption:

$$
\left\langle g^{2}\right\rangle_{\mathrm{coll}}=3\left\{1+\frac{1}{4} a_{2}\right\}, \quad\left\langle G^{2}\right\rangle_{\mathrm{coll}}=\frac{1}{2}\left\{1+\frac{1}{2} a_{2}\right\},
$$

$$
\begin{gathered}
\left\langle g^{* 2}\right\rangle_{\mathrm{coll}}=\left\langle g^{2}\right\rangle_{\mathrm{coll}}-\left(1-\alpha^{2}\right)\left\{1+\frac{1}{4} a_{2}\right\}, \\
\left\langle c_{1}^{2}\right\rangle_{\mathrm{coll}}=\left\langle G^{2}\right\rangle_{\mathrm{coll}}+\frac{1}{4}\left\langle g^{2}\right\rangle_{\mathrm{coll}}=\frac{5}{4}\left\{1+\frac{7}{20} a_{2}\right\}, \\
\left\langle\mathbf{c}_{1} \cdot \mathbf{c}_{2}\right\rangle_{\mathrm{coll}}=\left\langle G^{2}\right\rangle_{\mathrm{coll}}-\frac{1}{4}\left\langle g^{2}\right\rangle_{\mathrm{coll}}=-\frac{1}{4}\left\{1-\frac{1}{4} a_{2}\right\}, \\
\left\langle\mathbf{c}_{1}^{*} \cdot \mathbf{c}_{2}^{*}\right\rangle_{\mathrm{coll}}=\left\langle\mathbf{c}_{1} \cdot \mathbf{c}_{2}\right\rangle_{\mathrm{coll}}+\frac{1}{2}\left(1-\alpha^{2}\right)\left\{1+\frac{1}{4} a_{2}\right\} .
\end{gathered}
$$

Here, $\mathbf{c}_{i}^{*}$ are the postcollision velocities, as defined in paper I. The sum of the third and fourth equality depends only on the center-of-mass velocity, i.e., $\left\langle G^{2}\right\rangle_{\text {coll }}$. In the elastic limit $\alpha \rightarrow 1$, the average energy of a particle that is about to collide $\left\langle c^{2}\right\rangle_{\text {coll }}=(5 / 4)\left\langle c^{2}\right\rangle$, is above the mean energy per particle $\left\langle c^{2}\right\rangle$, which equals unity.

In the molecular chaos approximation, an average such as $\left\langle\left(\mathbf{c}_{1} \cdot \mathbf{c}_{2}\right)^{m} g^{n}\right\rangle_{\text {coll }}$ with $\{m, n\}$ integers, is in general nonvanishing, except in the special case $n=-1$. Then, $\left\langle\left(\mathbf{c}_{1} \cdot \mathbf{c}_{2}\right)^{m} / g\right\rangle_{\text {coll }}$ reduces to an unconstrained average, proportional $\left\langle\left(\mathbf{c}_{1} \cdot \mathbf{c}_{2}\right)^{m}\right\rangle$, which vanishes for odd values of $m$. Additional information about the relative orientation of the incoming velocities may be obtained from the distribution of the angle $\psi_{12}$, defined by $\mathbf{c}_{1} \cdot \mathbf{c}_{2}=c_{1} c_{2} \cos \psi_{12}$. A numerical calculation (again neglecting $a_{2}$ corrections) gives $\left\langle\cos \psi_{12}\right\rangle_{\text {coll }} \simeq-0.233$, which is close to the value -0.2 , estimated from $\left\langle\mathbf{c}_{1} \cdot \mathbf{c}_{2}\right\rangle_{\text {coll }} \simeq\left\langle c_{1}^{2}\right\rangle_{\text {coll }}\left\langle\cos \psi_{12}\right\rangle_{\text {coll }}$ using the above results.

A very sensitive probe for studying the violation of molecular chaos is the probability distribution $P(b)$ of impact parameters $b=|\hat{\mathbf{g}} \times \hat{\boldsymbol{\sigma}}|=\sin \theta$, where $\theta=\cos ^{-1}(\hat{\mathbf{g}} \cdot \hat{\boldsymbol{\sigma}})$ is the angle of incidence. It is defined as the collisional average

$$
P(b)=\langle\delta(b-|\hat{\mathbf{g}} \times \hat{\boldsymbol{\sigma}}|)\rangle_{\mathrm{coll}}=\frac{\int d \hat{\boldsymbol{\sigma}} \int d \mathbf{c}_{1} \int d \mathbf{c}_{2} \delta(b-|\hat{\mathbf{g}} \times \hat{\boldsymbol{\sigma}}|)|\mathbf{g} \cdot \hat{\boldsymbol{\sigma}}| \Theta(-\hat{\mathbf{g}} \cdot \hat{\boldsymbol{\sigma}}) f^{(2)}\left(\mathbf{c}_{1}, \mathbf{c}_{2}, \boldsymbol{\sigma}\right)}{\int d \hat{\boldsymbol{\sigma}} \int d \mathbf{c}_{1} \int d \mathbf{c}_{2}|\mathbf{g} \cdot \hat{\boldsymbol{\sigma}}| \Theta(-\hat{\mathbf{g}} \cdot \hat{\boldsymbol{\sigma}}) f^{(2)}\left(\mathbf{c}_{1}, \mathbf{c}_{2}, \boldsymbol{\sigma}\right)},
$$

and $P(b)$ can be easily computed in a molecular-dynamics experiment. As long as molecular chaos holds, the distribution of $b$ is independent of the functional form of $f$ and we obtain straightforwardly

$$
P(b)=\left\{\begin{array}{cc}
(d-1) b^{d-2} & \text { if } 0<b<1 \\
0 & \text { otherwise }
\end{array}\right.
$$

which reduces in two dimensions to the uniform distribution,

$$
P(b)= \begin{cases}1 & \text { if } 0<b<1 \\ 0 & \text { otherwise }\end{cases}
$$

In order to analyze molecular chaos breakdown in more detail, we have introduced a collection of moments $M_{n m}$ and their dimensionless counterparts $B_{n m}$ for $n, m=\{0,1,2 \ldots\}$, (see definition below), to analyze in detail the possible breakdown of the molecular chaos factorization (6). These moments $M_{n m}(T)$ of the pair distribution at contact are defined as,

$$
\begin{aligned}
M_{n m}(T) \equiv & \frac{2}{\Omega_{d}} \int d \mathbf{v}_{1} d \mathbf{v}_{2} \int d \hat{\boldsymbol{\sigma} \Theta} \\
& \times\left(-\mathbf{v}_{12} \cdot \hat{\boldsymbol{\sigma}}\right) \hat{f}^{(2)}\left(\mathbf{v}_{1}, \mathbf{v}_{2}, \boldsymbol{\sigma} \mid T\right) v_{12}^{n}|\cos \theta|^{m},
\end{aligned}
$$


which are averages over the precollision hemisphere, where $\theta=\cos ^{-1}(\hat{\mathbf{g}} \cdot \hat{\boldsymbol{\sigma}})$. Let $M_{n m}^{\mathrm{E}}\left(T_{\mathrm{E}}\right)$ denote the same quantity evaluated in Enskog's formulation of the molecular chaos approximation, and evaluated at the reference temperature $T_{\mathrm{E}}$, i.e., evaluated with $\hat{f}^{(2)}\left(\mathbf{v}_{1}, \mathbf{v}_{2}, \boldsymbol{\sigma} \mid T\right)$ replaced by $\chi_{\mathrm{E}} \hat{f}\left(\mathbf{v}_{1} \mid T_{\mathrm{E}}\right) \hat{f}\left(\mathbf{v}_{2} \mid T_{\mathrm{E}}\right)$, then the reduced moments are defined as

$$
B_{n m}(T)=M_{n m}(T) / M_{n m}^{\mathrm{E}}\left(T_{\mathrm{E}}\right)
$$

where $M_{n m}^{\mathrm{E}}\left(T_{\mathrm{E}}\right)$ is evaluated in Eq. (A2). It is proportional to $v_{\mathrm{E}}^{n}$, where $v_{\mathrm{E}}=\sqrt{2 T_{\mathrm{E}} / m}$. We prefer to normalize the reduced moments by $M_{n m}^{\mathrm{E}}\left(T_{\mathrm{E}}\right)$, because its analytic form is given explicitly. One could also normalize by $M_{n m}^{\mathrm{E}}(T)$ $=\left(T_{\mathrm{E}} / T\right)^{n / 2} M_{n m}^{\mathrm{E}}\left(T_{\mathrm{E}}\right)$. The disadvantage of $M_{n m}^{\mathrm{E}}(T)$ is that the computation requires the simulated values of the kinetic temperature $T$. The collisional averages $\left\langle v_{12}^{n}|\cos \theta|^{m}\right\rangle_{\text {coll }}$ expressed in terms of these new moments give

$$
\left\langle v_{12}^{n}|\cos \theta|^{m}\right\rangle_{\mathrm{coll}}=\frac{M_{n+1, m+1}(T)}{M_{11}(T)} .
$$

We first observe that the average collision frequency $\omega$, defined in Eq. (5), is proportional to $M_{11}(T)$, so that

$$
B_{11}(T)=\frac{M_{11}(T)}{M_{11}^{\mathrm{E}}\left(T_{\mathrm{E}}\right)}=\frac{\omega}{\omega_{\mathrm{E}}}
$$

with $\omega_{\mathrm{E}}$ defined in Eq. (19). This implies that the reduced moments $B_{n m}(T)$ may all be expressed in collisional averages, i.e.,

$$
B_{n m}(T)=\frac{\omega}{\omega_{\mathrm{E}}} \frac{\left\langle v_{12}^{n-1}|\cos \theta|^{m-1}\right\rangle_{\mathrm{coll}}}{\left\langle v_{12}^{n-1}|\cos \theta|^{m-1}\right\rangle_{\mathrm{coll}}^{\mathrm{E}}} .
$$

The average $\langle\cdots\rangle_{\text {coll }}^{\mathrm{E}}$ is defined through Eq. (30) with $M_{n m}(T)$ replaced by $M_{n m}^{\mathrm{E}}\left(T_{\mathrm{E}}\right)$, and calculated in Eq. (A2). It represents the collisional average, evaluated with the Enskog factorization $f^{(2)}=\chi_{\mathrm{E}}$ ffand also taken at the reference temperature $T_{\mathrm{E}}$. Note that the equality (32) consists of two factors, $\omega$ and $\langle\cdots\rangle_{\text {coll }}$, which are measured separately in event driven MD simulations.

We also observe that the equality $\Gamma(T)=\Gamma_{\mathrm{E}}\left(T_{\mathrm{E}}\right)$, explained above Eq. (18), implies that

$$
B_{33}(T)=\frac{M_{33}(T)}{M_{33}^{\mathrm{E}}\left(T_{\mathrm{E}}\right)}=\frac{\Gamma(T)}{\Gamma^{\mathrm{E}}\left(T_{\mathrm{E}}\right)}=1 .
$$

Furthermore, we have for the excess pressure $p^{\mathrm{ex}}(T)$ $\equiv p(T)-n T$,

$$
B_{22}(T)=\frac{p^{\mathrm{ex}}(T) / n T}{p_{\mathrm{E}}^{\mathrm{ex}}\left(T_{\mathrm{E}}\right) / n T_{\mathrm{E}}}=\frac{\omega}{\omega_{\mathrm{E}}} \times \frac{\left\langle\left|v_{12} \cos \theta\right|\right\rangle_{\mathrm{coll}}}{\left\langle\left|v_{12} \cos \theta\right|\right\rangle_{\mathrm{coll}}^{\mathrm{E}}}
$$

and for the dynamic pair correlation at contact $\chi^{(-)}$,

$$
B_{00}(T)=\frac{\chi^{(-)}}{\chi_{\mathrm{E}}}=\frac{\left\langle\left|v_{12} \cos \theta\right|^{-1}\right\rangle_{\text {coll }}}{\left\langle\left|v_{12} \cos \theta\right|^{-1}\right\rangle_{\text {coll }}^{\mathrm{E}}} .
$$

In the Appendix, we present a more complete set of relations for the $B_{n m}$.

In the next section MD simulations will show that the predicted deviation from a Maxwellian [see Eq. (2)] in the (unconstrained) velocity distribution of a single particle may be observed for small inelasticities. However, larger deviations are found between the observed constrained probability distributions and averages, and the corresponding kinetic theory predictions given by Eq. (24), based on molecular chaos. Consequently, the small corrections resulting from $a_{2}$ in Eq. (2) may be neglected in most cases.

\section{Radial distributions}

The static or unconstrained radial distribution function in the spatially homogeneous IHS fluid is defined as

$$
g(r)=\int \frac{\mathrm{d} \hat{\boldsymbol{\sigma}}}{\Omega_{d}} \int \mathrm{d} \mathbf{c}_{1} \mathrm{~d} \mathbf{c}_{2} f^{(2)}\left(\mathbf{c}_{1}, \mathbf{c}_{2}, r \hat{\boldsymbol{\sigma}}\right) .
$$

It may be averaged over all directions of $\mathbf{r}$ because of statistical isotropy. The unconstrained radial distribution function at contact is defined as the extrapolation, $Y=g(r \rightarrow \sigma+0)$. By splitting the $\hat{\boldsymbol{\sigma}}$ integration into a precollision $(\hat{\mathbf{g}} \cdot \hat{\boldsymbol{\sigma}}<0)$ and postcollision hemisphere $(\hat{\mathbf{g}} \cdot \hat{\boldsymbol{\sigma}}>0)$, we obtain $Y$ as sum of two terms,

$$
Y=\frac{1}{2}\left(Y^{(-)}+Y^{(+)}\right) .
$$

The definitions of $Y^{(-)}, Y^{(+)}$follow from Eq. (36) by adding, respectively, factors $\Theta(-\hat{\mathbf{g}} \cdot \hat{\boldsymbol{\sigma}})$ and $\Theta(\hat{\mathbf{g}} \cdot \hat{\boldsymbol{\sigma}})$ under the integral sign in Eq. (36). The dummy integration variables in $Y^{(+)}$represent the postcollision velocities, $\left(\mathbf{c}_{1}^{*}, \mathbf{c}_{2}^{*}\right)$, corresponding to the precollision ones, $\left(\mathbf{c}_{1}, \mathbf{c}_{2}\right)$.

On the other hand, we have the dynamic precollision correlation $\chi^{(-)}$, defined in Eq. (8), and a similar postcollision one, $\chi^{(+)}$, defined by replacing $\Theta(-\hat{\mathbf{g}} \cdot \hat{\boldsymbol{\sigma}})$ in Eq. (8) by $\Theta(\hat{\mathbf{g}} \cdot \hat{\boldsymbol{\sigma}})$. They are related by continuity of flux. Because the incident flux of $\left(\mathbf{c}_{1} \mathbf{c}_{2}\right)$ pairs just before collision is equal to the scattered flux of $\left(\mathbf{c}_{1}^{*} \mathbf{c}_{2}^{*}\right)$ pairs just after collision, we have inside dynamic averages the equality

$$
\begin{aligned}
& \Theta\left(-g_{n}\right)\left|g_{n}\right| f^{(2)}\left(\mathbf{c}_{1} \mathbf{c}_{2}, \boldsymbol{\sigma}\right) \mathrm{d} \mathbf{c}_{1} \mathrm{~d} \mathbf{c}_{2} \mathrm{~d} \hat{\boldsymbol{\sigma}} \\
& =\Theta\left(g_{n}^{*}\right)\left|g_{n}^{*}\right| f^{(2)}\left(\mathbf{c}_{1}^{*} \mathbf{c}_{2}^{*}, \boldsymbol{\sigma}\right) \mathrm{d} \mathbf{c}_{1}^{*} \mathrm{~d} \mathbf{c}_{2}^{*} \mathrm{~d} \hat{\boldsymbol{\sigma}},
\end{aligned}
$$

where $g_{n}=\mathbf{g} \cdot \hat{\boldsymbol{\sigma}}=g \cos \theta$. The reflection law $g_{n}^{*}=\alpha\left|g_{n}\right|$, for inelastic collisions in combination with the continuity of the flux and Eq. (8) yields at once,

$$
\chi^{(+)}=(1 / \alpha) \chi^{(-)} .
$$

In principle, Eqs. (37) and (20) provide two alternative routes to compute the precollisional pair correlation at con- 
tact: the first one, denoted by $Y^{(-)}$, may be implemented numerically as a static or unconstrained average, namely, by extrapolation to contact of the pair-correlation function for pairs aiming to collide. The second one, denoted by $\chi^{(-)}$, may be computed as a dynamic collisional average, calculated from $f^{(2)}\left(\mathbf{c}_{1}, \mathbf{c}_{2}, \boldsymbol{\sigma}\right)$ at contact. It is important to stress that the dynamic $\chi^{(-)}$is calculated as a time average over the subset of colliding pairs at contact, and the static $Y^{(-)}$as a time average over all pairs, satisfying the relation, $\mathbf{g} \cdot \hat{\boldsymbol{\sigma}}$ $<0$ and extrapolated to $r \rightarrow \sigma+0$, i.e., calculated from $f^{(2)}\left(\mathbf{c}_{1}, \mathbf{c}_{2}, \mathbf{r}\right)$, where the limit is taken after all integrations have been performed. This may lead to different results, because the integrand contains the the function $f^{(2)}$ that turns out to be singular near $r=\sigma$ and $\mathbf{v}_{12}$ small (see discussion in Sec. III C).

Physically, it is also clear why the averages in the NESS need not be the same. For instance, the relation (38) may not hold for the limiting $(r \rightarrow \sigma)$ values, $Y^{(-)}$and $Y^{(+)}$, because non-mean-field effects (in particular, the "rotation-induced" recollisions discussed at the start of Sec. III, or noise-induced recollisions, see below) may result in differences between the two methods to evaluate $\chi^{(-)}$and $Y^{(-)}$. The reason is that the validity of Eq. (38), expressing flux continuity for the limiting values $(r \rightarrow \sigma+0)$, is questionable in the presence of the external random force. When the kicking frequency is much larger than the collision frequency (situation considered here), a pair of particles may indeed be put in contact under the action of the random force only. We will investigate possible numerical differences between $\chi^{(-)}$and $Y^{(-)}$in the next section on MD simulations.

In paper I, we have studied the long-range spatial correlation functions $G_{a b}(r)$ of the density field $n(\mathbf{r}, t)$ and the flow field $\mathbf{u}(\mathbf{r}, t)$ in the NESS. These functions are closely related to Eq. (36), i.e.,

$$
\begin{aligned}
G_{n n}(r) & =\frac{1}{n^{2}}\left\langle\sum_{i} \delta\left(\mathbf{r}_{i}-\mathbf{r}\right)\left[\sum_{j} \delta\left(\mathbf{r}_{j}\right)-n\right]\right\rangle \\
& =\frac{1}{n} \delta(\mathbf{r})+(g(r)-1)
\end{aligned}
$$

and, in the notation of paper I,

$$
\begin{aligned}
G_{\mathbf{u u}}(r) & =\frac{1}{n^{2}}\left\langle\sum_{i, j} \mathbf{v}_{i} \cdot \mathbf{v}_{j} \delta\left(\mathbf{r}_{i}-\mathbf{r}\right) \delta\left(\mathbf{r}_{j}\right)\right\rangle \\
& =G_{\|}(r)+(d-1) G_{\perp}(r) \\
& =\frac{d}{2} \frac{v_{0}^{2}}{n} \delta(\mathbf{r})+v_{0}^{2} g(r)\left\langle\mathbf{c}_{1} \cdot \mathbf{c}_{2}\right\rangle(r),
\end{aligned}
$$

where $\langle\cdots\rangle$ is an average over the $N$-particle nonequilibrium steady state and the static velocity correlation, $\left\langle\mathbf{c}_{1} \cdot \mathbf{c}_{2}\right\rangle(r)$, is defined as

$$
\left\langle\mathbf{c}_{1} \cdot \mathbf{c}_{2}\right\rangle(r)=\int \frac{\mathrm{d} \hat{\boldsymbol{\sigma}}}{\Omega_{d}} \int \mathrm{d} \mathbf{c}_{1} \mathrm{~d} \mathbf{c}_{2} f^{(2)}\left(\mathbf{c}_{1}, \mathbf{c}_{2}, r \hat{\boldsymbol{\sigma}}\right)\left(\mathbf{c}_{1} \cdot \mathbf{c}_{2}\right) / g(r) .
$$

The correlation functions $G_{a b}(r)$ above are very long ranged, decaying similar to $r^{2-d}$ for large distances. In the first part of this section, we have introduced the static correlations, $Y, Y^{( \pm)}$, and the dynamic ones, $\chi^{( \pm)}$. In Eq. (A8) and (A9) we have done the same for the dynamic counter parts $\left\langle\mathbf{c}_{1} \cdot \mathbf{c}_{2}\right\rangle_{\text {dyn }}$ and $\left\langle\mathbf{c}_{1} \cdot \mathbf{c}_{2}\right\rangle_{\text {dyn }}^{(-)}$of the static correlation $\left\langle\mathbf{c}_{1} \cdot \mathbf{c}_{2}\right\rangle(r$ $\rightarrow \sigma$ ), introduced in Eq. (41).

In the next section, the short-range behavior of these functions will be studied by MD simulations.

\section{SIMULATION RESULTS FOR THE NESS}

To investigate the short-scale structure characterizing the NESS and the validity of molecular chaos, we will present in this section MD simulation results, and compare these with our theoretical predictions whenever possible. The details of the simulations of the randomly driven inelastic hard disk system have been reported elsewhere [10]. We will work in the limit in which the kicking frequency of the external random force is much larger than the collision frequency. This is the limit in which the Fokker-Planck term in Eq. (1) models the random energy input through the random kicks. The external random force will, in principle, have a quantitative influence on the short range structure of the fluid. There is only one important difference with respect to the simulations carried out in [10]. There, the random rotation proposed in [36] was implemented to avoid inelastic collapse at high inelasticities $(\alpha<0.5)$. This procedure amounts to rotating the relative velocity $\mathbf{g}$ by a small random angle after each collision. Consider the completely inelastic situation $\alpha=0$ for the sake of the argument. After each collision, the vector $\mathbf{g}$ lies exactly at the border of the precollisional hemisphere $(\mathbf{g} \cdot \hat{\boldsymbol{\sigma}}$ $=0$ ), so that if the aforementioned random angle has equiprobable positive and negative values, the rotation procedure will lead to a recollision with probability $1 / 2$. This leads to a spurious increase of the number of collisions by a factor $\sum_{n=0}^{\infty} 1 / 2^{n}=2$ (the recollision may itself induce a recollision with probability $1 / 2$ etc. so that the frequency of collision effectively doubles). When $\alpha$ is small but nonvanishing, this effect is still present but weaker. This is clearly an artificial violation of molecular chaos that has been discarded in the present paper: for $\alpha<0.5$, we have also implemented the rotation procedure, but if a small rotation leads to a recollision, a new angle is drawn until the pair separates. In this way, we reduce an important source of correlations (the effect is dramatic on all the low-order moments $B_{n m}$, not only on the collision frequency; in particular, the moments with $n \leqslant 1$ that correspond to collisional averages of negative powers of $g$, are strongly biased toward bigger values if the "rotation-induced recollisions" are present). After applying this rule, we are then left only with correlations induced by the hard sphere dynamics plus the ones induced by the noise itself (see below). 


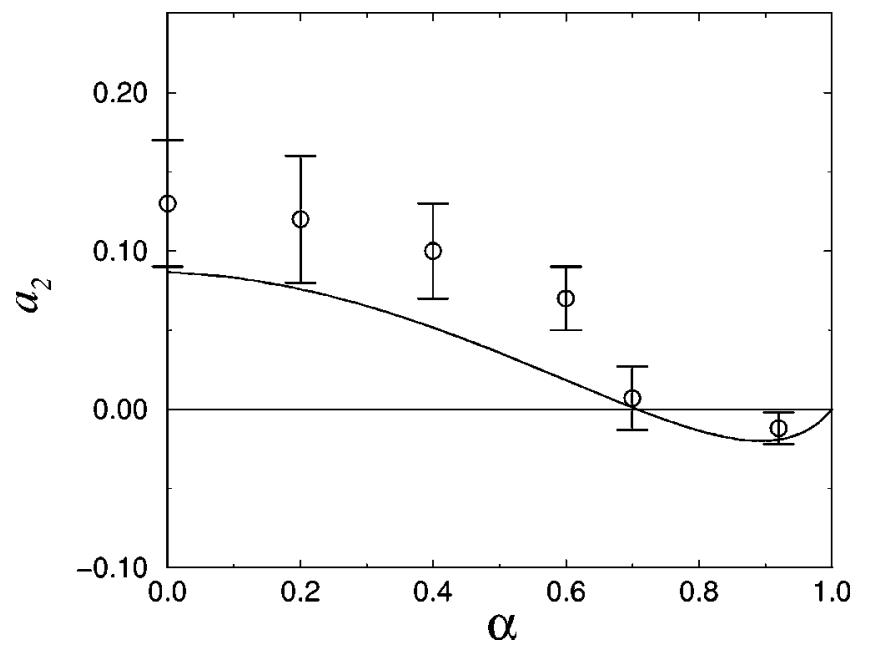

FIG. 2. Fourth cumulant as a function of the coefficient of restitution. Comparison is made between the two-dimensional version of Eq. (4) and MD results (circles) obtained for a system of 10201 inelastic disks, measured at several densities (see Sec. III A).

\section{A. Cumulants}

First, we focus on the single-particle velocity distribution function $f$ averaged over all particles, which deviates from a Maxwellian distribution due to the inelasticity of the collisions. In the previous section, we presented predictions for these deviations, assuming molecular chaos. The resulting expression given by Eq. (4) for the fourth cumulant $a_{2}$ of the distribution as well as the prediction for its overpopulated tail are in perfect agreement with three-dimensional (3D) direct simulation monte carlo (DSMC) results over the whole region of inelasticities [34]. As DSMC itself invokes molecular chaos, this observation merely justifies the approximations made in the analytic calculation. Information about the validity of molecular chaos may only be obtained from a comparison with molecular- dynamics simulations, and Fig. 2 shows this comparison for the fourth cumulant $a_{2}$ as a function of the coefficient of restitution $\alpha$ in 2D. The simulation results are in agreement with Eq. (4) for small inelasticity, but start to deviate significantly from the theoretical prediction at $\alpha=0.6$. These deviations, together with the perfect agreement between the theoretical prediction and DSMC results, provide direct evidence for the breakdown of molecular chaos for $\alpha \lesssim 0.6$. The theoretical result (4) is independent of the density. As $a_{2}$ represents only a small correction in Eq. (2), one needs a large number of collisions and a large number of particles to reach sufficient statistical accuracy. So, high densities, for which one may use linked lists [30], are well suited. The data in Fig. 2 are typically obtained at high- packing fractions ( $\phi=0.63$ at $\alpha=0.92$ and 0.7 , and $\phi=0.55$ at $\alpha=0.6)$ and $N=10201$ particles. At low densities and weaker inelasticities ( $\alpha \gtrsim 0.6)$ we are unable to collect enough statistics to measure the small correction, represented by $a_{2}$. Simulations at higher inelasticities did not show any density dependence of $a_{2}$ in the range $0.2 \lesssim \phi$ $\lesssim 0.6$, suggesting that the cumulant expression (3), obtained from the Enskog-Boltzmann equation also applies at liquid

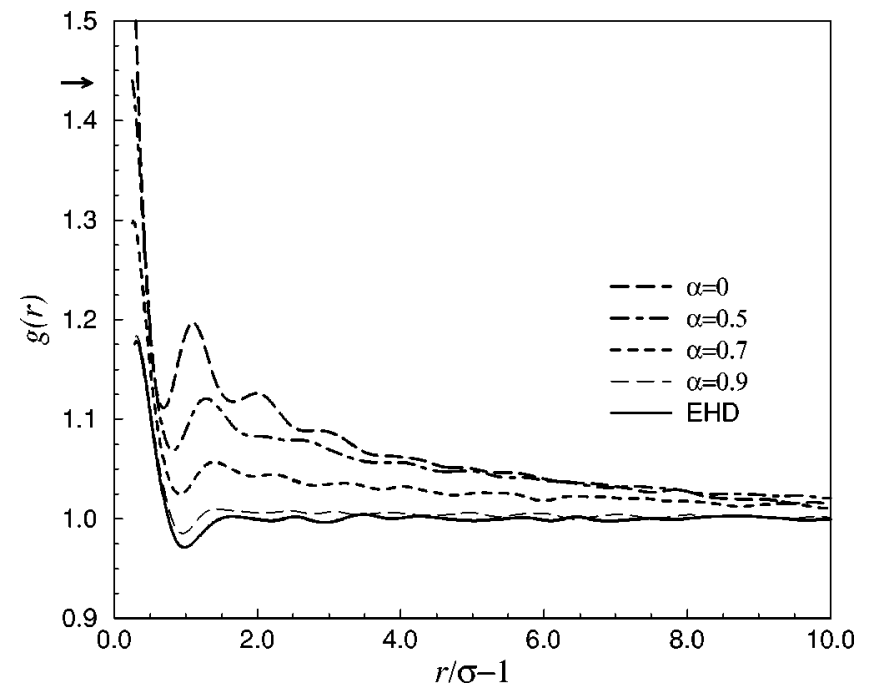

FIG. 3. Pair-distribution functions $g(r)$ versus distance between the particles at a packing fraction $\phi=0.2$. The arrow indicates the value at contact for an elastic hard disc (EHD) fluid (from Verlet and Levesque [31]).

densities. Similar results as those displayed in Fig. 2 have been observed for the 3D version of the present model [11].

\section{B. Radial distribution function}

Next, we present results for the static or unconstrained radial distribution function $g(r)$, and in particular, its extrapolated values at contact $Y, Y^{(-)}, Y^{(+)}$. Here, $g(r)$ is essentially the density-density correlation function, whose long range behavior was studied in Ref. [10].

Figure 3 shows the measured values of $g(r)$ for short distances and packing fraction $\phi=0.2$, at different inelasticities. At small inelasticities $(\alpha \simeq 0.9), g(r)$ resembles the radial distribution function for elastic hard disks (EHD). At higher inelasticities, deviations start to appear: the first and second maximum in the measured $g(r)$ are enhanced with respect to their EHD values at the same density. Moreover, the functional shape also deviates from the corresponding pair distribution of EHD at an appropriately chosen higher density; e.g., if this density is chosen such that the value of the second maximum of the pair distribution of EHD coincides with the simulation result for IHD, the observed value at contact would still be underestimated by the EHD pair distribution.

It seems worthwhile to compare these results with existing experiments on granular fluids in which the pair distribution $g(r)$ has been measured. In the experiment of Ref. [2] on a vertically vibrated thin granular layer, $g(r)$ has been measured at $\phi=0.46$. In the fluidized ("gaslike") phase, it follows the equilibrium result for elastic hard disks almost identically. This result may be compared to our simulations for a randomly driven fluid of inelastic disks at $\alpha=0.9$, corresponding to the value for stainless steel balls used in the experiment. It would be of interest to measure experimentally how $g(r)$ in the fluidized phase depends on the inelasticity, and see if a behavior similar to that of Fig. 3 is observed. It is also interesting to note that the pair-correlation 

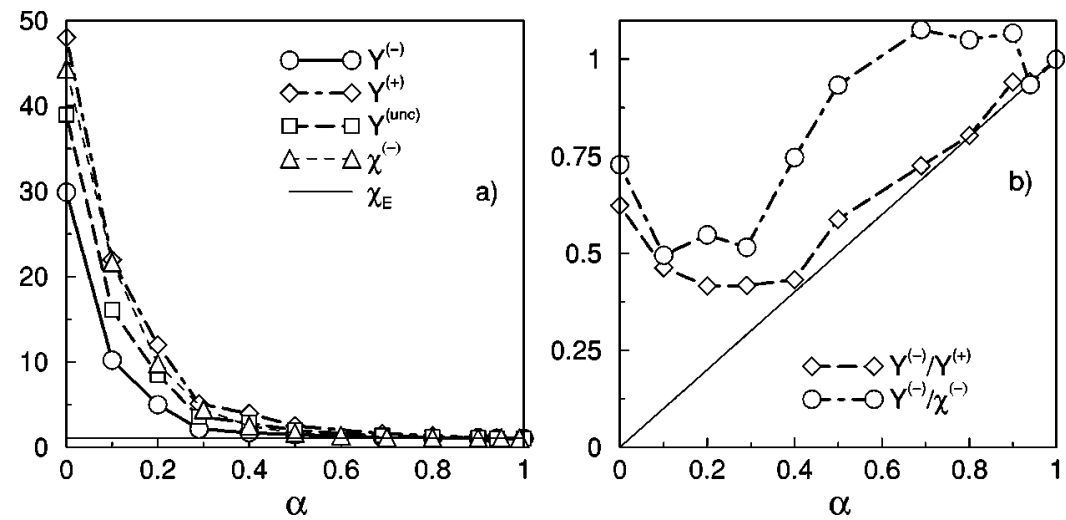

FIG. 4. (a) Static or unconstrained pair-distribution functions at contact $Y, Y^{(-)}, Y^{(+)}$, as extrapolated from the corresponding pairdistribution function $g(r \rightarrow \sigma)$ at $\phi=0.05$, compared with the dynamic correlation $\chi^{(-)}$at contact. The straight line corresponds to the EHD prediction $\left(\chi_{\mathrm{E}}=1.084\right)$. (b) Ratio of dynamic to static correlation $\chi^{(-)} / Y^{(-)}$, to be compared with 1 , and the static ratio $Y^{(-)} / Y^{(+)}$, to be compared with the dynamic ratio $\chi^{(-)} / \chi^{(+)}=\alpha$.

function $g(r)$ in a non-Brownian suspension of spherical particles, fluidized between two vertical parallel plates, shows an enhanced value at contact as well [35].

In Fig. 4(a), we show the value at contact, $Y$, obtained by extrapolation from $g(r)$ at $\phi=0.05$, together with the extrapolated values for approaching and receding pairs, $Y^{(-)}$ and $Y^{(+)}$, respectively. For $\alpha \gtrsim 0.8$, no significant deviations are found from the Verlet-Levesque value $\chi_{\mathrm{E}}=1.084$ for elastic hard disks at the same density. More surprising is the value of $g(r=\sigma)$ at large inelasticities, reaching a value around 40 for $\alpha \rightarrow 0$. This property, combined with the observation that the first and second maximum in $g(r)$ are shifted to smaller $r$ values, and are larger (up to $20 \%$ at small $\alpha$ ) than the corresponding hard disks values, may be interpreted as a tendency to cluster, i.e., to stay in continuously rearranging configurations with large-density inhomogeneities. We return to this point in Sec. III G.

Figure 4 also shows the dynamic correlation, $\chi^{(-)}$ $=\chi_{\mathrm{E}} B_{00}$, measured as a collisional average. Figure 4(b) compares the static ratio $Y^{(-)} / Y^{(+)}$with the dynamic one, $\chi^{(-)} / \chi^{(+)}=\alpha$, and also shows the ratio $Y^{(-)} / \chi^{(-)}$. The plots clearly show that the dynamic and static correlations $\chi^{( \pm)}$ and $Y^{( \pm)}$are different. For $\alpha \lesssim 0.5$, the differences are large, and for $\alpha \gtrsim 0.6$, both functions are about equal. For the case of a freely evolving IHS fluid, Soto and Maréschal [18] have recently observed a similar behavior, and explained it in terms of the effect of the increase of grazing collisions on the effective $\chi^{(-)}$. In the randomly driven IHD fluid the same effects are present. All correlations, $\chi^{(-)}, Y^{( \pm)}$are large, especially at small $\alpha$. This is caused by the divergence of $f^{(2)}\left(\mathbf{c}_{1}, \mathbf{c}_{1}, \boldsymbol{\sigma}\right)$ at small $g$ and small $\cos \theta$, which corresponds to grazing collisions and will be further discussed in the next section. As a result of noise-induced recollisions, collisions with small $g$ and small $\cos \theta$ are oversampled; consequently, a dynamical average involving negative powers of $g \cos \theta$ such as $\chi^{(-)}$is expected to be larger than its static counterpart $Y^{(-)}$. This feature may be observed in Fig. 4(b). Moreover, in the absence of recollisions, we would expect $Y^{(-)}$ $=\alpha Y^{(+)}$as a result of plain hard-sphere dynamics. However, in the heated system, the flux continuity, as expressed in Eq.
(38), is no longer satisfied for the extrapolated $Y$ 's; some pairs are put in precollision configuration under the action of the random force, which leads to $Y^{(-)}>\alpha Y^{(+)}$. The breakdown of $Y^{(-)}=\alpha Y^{(+)}$signals the inelasticity beyond which noise-induced correlations become relevant. It is furthermore possible to consider situations where the recollisions dominate the dynamics, e.g., at small $\alpha$ by allowing rotationinduced recollisions. In this extreme limit, we expect $Y^{(-)}$ $\simeq Y^{(+)}$, the pairs being put in pre- or post-collision configuration essentially at random. In the same limit, the population of colliding pairs with small $g$ and small $\cos \theta$ is enhanced, leading to a more pronounced discrepancy between dynamic and static averages [i.e., a much smaller ratio $Y^{(-)} / \chi^{(-)}$than observed in Fig. 4(b)].

\section{Equation of state. Molecular chaos breakdown}

To what extent does the extrapolated static radial distribution function, $Y=g(r \rightarrow \sigma)$ describe the nontrivial dependence in the NESS of collision frequency in Eq. (5), collisional damping in Eq. (14) and pressure in Eq. (12) on the inelasticity? If molecular chaos holds, the latter quantities depend, according to Eqs. (6), (13),(9), and (15), on the precollisional pair function at contact, $\chi^{(-)}$, where the particles are aiming to collide. This function differs from the extrapolated static $Y^{(-)}$at high inelasticities (see Fig. 4). Consider first the collision frequency in the molecular chaos approximation, $\omega_{\mathrm{mc}}=\chi \omega_{0}(T)$ above Eq. (9), with $\chi=\chi^{(-)}$the dynamic correlation, i.e.,

$$
\frac{\omega_{\mathrm{mc}}(T)}{\omega_{\mathrm{E}}}=\frac{\chi^{(-)}}{\chi_{\mathrm{E}}} \frac{v_{0}}{v_{\mathrm{E}}}=B_{00} \sqrt{\frac{T}{T_{\mathrm{E}}}},
$$

where we have used Eqs. (9) and (35). This is an extremely poor approximation, as can be seen from Fig. 1, which shows that the measured value $\omega / \omega_{\mathrm{E}}$ approaches 5.6 as $\alpha \rightarrow 0$, whereas $B_{00}$ is essentially divergent. Next, we replace $\chi^{(-)}$ in Eq. (43) by its static counterpart $Y^{(-)}$, shown in Fig. 4. This yields $\omega_{\text {stat }}(T) / \omega_{\mathrm{E}}=Y^{(-)} v_{0} / \chi^{(-)} v_{\mathrm{E}}$. Its limiting value for $\alpha \rightarrow 0$ is about a factor three too large when compared to 


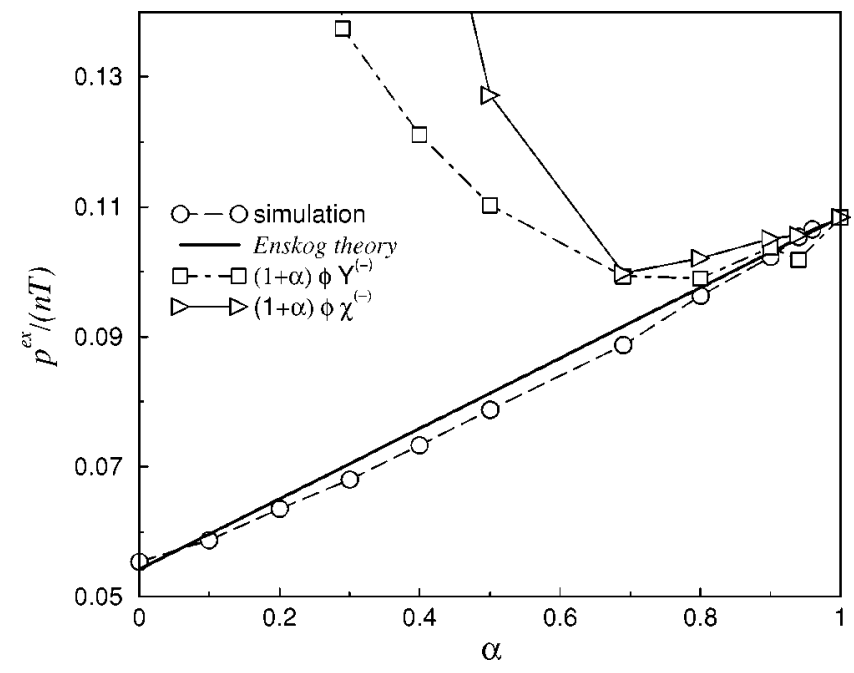

FIG. 5. Pressure versus coefficient of restitution at a packing fraction $\phi=0.05$. The simulations results (direct or through $B_{22}$ ) are compared with molecular chaos prediction (13), where $\chi$ is either the static $Y^{(-)}$in Eq. (37), or the dynamic $\chi^{(-)}$in Eq. (8), or the Enskog approximation $\chi_{\mathrm{E}}$ in Eq. (7), corresponding to $B_{22}=1$.

$\omega / \omega_{\mathrm{E}}$. We conclude that all mean-field approximations for the collision frequency, including the Enskog approximation $\omega_{\mathrm{E}}(T) / \omega_{\mathrm{E}}=\sqrt{T / T_{\mathrm{E}}}$, break down for $\alpha<0.6$.

Figure 5 shows the pressure of the IHD fluid, compared with the molecular chaos prediction given by Eq. (13), taking for $\chi$ either the Enskog approximation $\chi_{\mathrm{E}}$ in Eq. (7), or the simulated $Y^{(-)}$, or $\chi^{(-)}$. The Enskog approximation, accounting for the short-range geometric exclusion effects in the precollision state, gives a reasonable description of $p(T)$ for all $\alpha$, while both the static $Y^{(-)}$and the dynamic $\chi^{(-)}$ give an extremely poor description except for $\alpha>0.8$.

Consistent with this conclusion is the good estimate for the temperature $T_{\mathrm{E}}$ in the NESS, obtained by balancing the energy dissipation rate $\Gamma_{\mathrm{E}}\left(T_{\mathrm{E}}\right)$ in Eq. (16) with the energy input from the random force, as shown in Fig. 1. Moreover, the collisional energy loss $\Gamma(T) / \Gamma_{\mathrm{E}}(T)=\left(T_{\mathrm{E}} / T\right)^{3 / 2}$ in Eq. (18), is in agreement with MD simulations over the whole $\alpha$ interval within $30 \%$. All other mean-field approximations with $\omega_{\mathrm{E}}$ replaced by $\omega_{\mathrm{mc}}(T)$ or $\omega_{\text {stat }}(T)$ give very poor results for $\Gamma(T)=m \xi_{0}^{2}$.

How can these paradoxical results be reconciled? Let us compare the individual definitions of $\chi^{(-)}, \omega, p$, and $\Gamma$, which all contain factors $|\mathbf{g} \cdot \boldsymbol{\sigma}|^{n} f^{(2)}\left(\mathbf{c}_{1}, \mathbf{c}_{2}, \boldsymbol{\sigma}\right)$ with $n$ $=0,1,2,3$. To find a possible explanation of these paradoxical results, we test the following scenario: the molecular chaos assumption (6) only breaks down at very small relative velocities $\mathbf{g}$, and more precisely, at very small $g_{n}=\mathbf{g} \cdot \hat{\boldsymbol{\sigma}}$ $=g \cos \theta$, which is the component of $\mathbf{g}$, parallel to the line of centers of the colliding particles (physical arguments for this scenario will be offered in Sec. III F where we discuss the noise-induced recollisions). On the basis of this scenario, the singularity in $f^{(2)}$ at small $\mathbf{g}$ makes the dynamic correlation $\chi^{(-)}=B_{00} \chi_{\mathrm{E}}$ (shown in Fig. 6) very much larger than $\chi_{\mathrm{E}}$, essentially divergent as $\alpha \rightarrow 0$. In calculating the collisional frequency from Eq. (5), the extra factor $g_{n}$ in the integrand

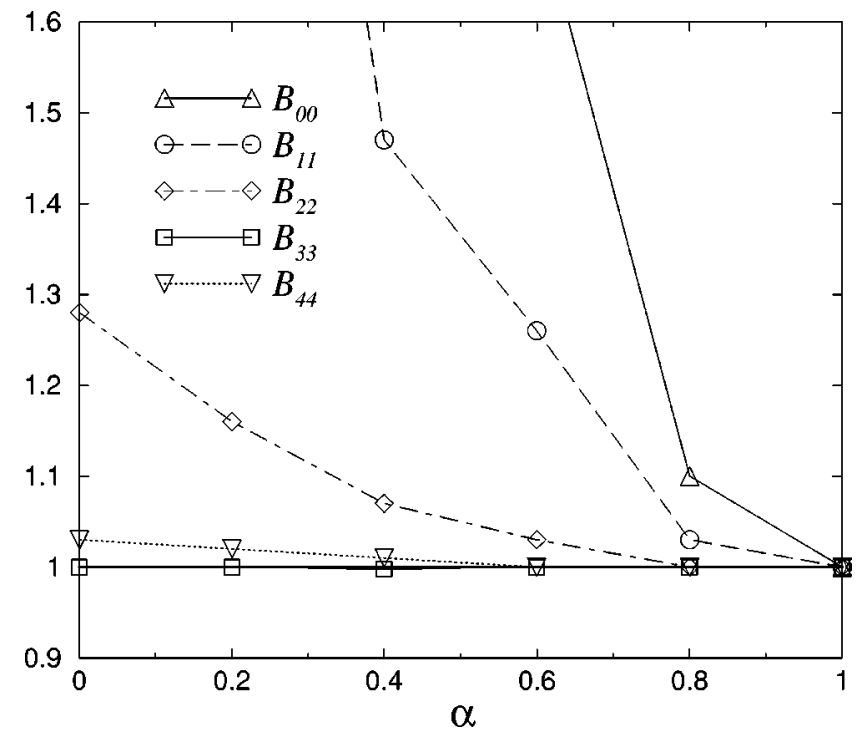

FIG. 6. Reduced moments $B_{n n}$ for $n=1,2,3$ as a function of the restitution coefficient at $\phi=0.2$ and $N=5041$. A similar behavior is observed at lower densities.

makes the small $g_{n}$ singularity integrable, giving a finite correction to the Enskog collision frequency, also for $\alpha \rightarrow 0$ (see Fig. 1). The contributions of the small $g_{n}$-singularity in $p(T)$ and $\Gamma(T)$ are essentially suppressed by extra factors of $|\mathbf{g} \cdot \hat{\boldsymbol{\sigma}}|^{n}$.

This possibility has been analyzed systematically by measuring the behavior of the moments $B_{n m}(T)$, which are useful tools to investigate the breakdown of the molecular chaos assumption. We have made $n$ and/or $m$ small in order to analyze the nature of the singularities in $f^{(2)}$ near small relative velocities and near grazing collisions, as displayed in Fig. 7. All deviations of these quantities from unity give a quantitative measure for the violation of the molecular chaos assumption. In the elastic limit, we have carefully checked for a large number of cases that the reduced moments $B_{n m}$ tend to one. Figure 6 shows the values of different moments $B_{n n}(T)$, and one can clearly see how the deviations from the elastic limit rapidly decrease as $n$ increases to $n=3$, after which they start to increase slowly. For larger $n$-values, the moments are reasonably close to unity, but statistical inaccuracy precludes any definite conclusion about the large $n$ behavior.

Further evidence for the above scenario is shown in Fig. 7 , where we display two sequences of moments $B_{n m}$. To draw some further conclusions from Figs. 6 and 7, we note that the integrands in $B_{n n}, B_{0 n}$, and $B_{n 0}$, as defined in Eq. (28), contain apart from $f^{(2)}$, respectively, the factors $g^{n}|\cos \theta|^{n},|\cos \theta|^{n}, g^{n}$. The reduced moments $B_{01}$ and $B_{10}$ contain again very large contributions from the divergence of $f^{(2)}$ near vanishing $g_{n}=g \cos \theta$. Figure 7 suggests that the presence of equal powers of $g$ and $\cos \theta$ in $B_{n n}$ simultaneously suppresses the large contributions from the singularities at $g=0$ and $\cos \theta=0$.

We conclude that the numerical results, displayed in Figs. 6 and 7, give support to the previous scenario, showing that molecular chaos breaks down only in a very small portion of 

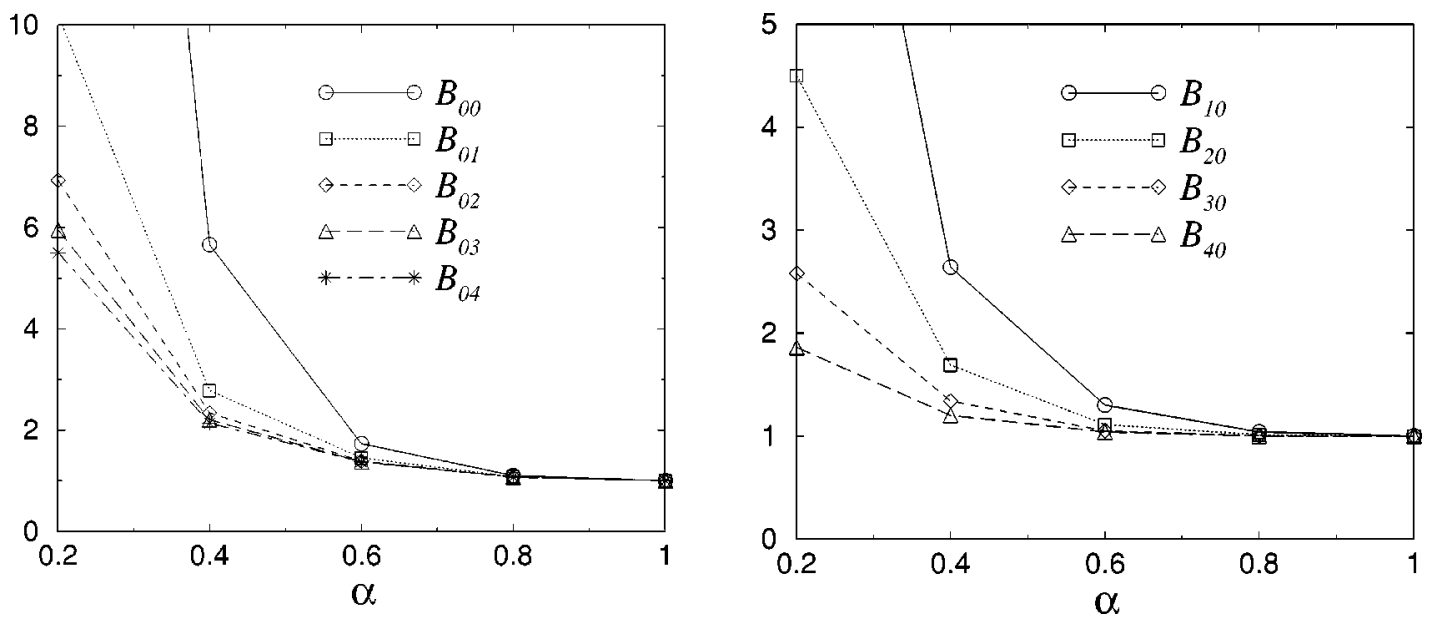

FIG. 7. Reduced moments $B_{0 m}$ and $B_{n 0}$ as a function of $\alpha$ at $\phi=0.2$ and $N=5041$.

the phase space, around $g_{n}=\mathbf{g} \cdot \hat{\boldsymbol{\sigma}}=g \cos \theta=0$. The size of this "pocket" in phase space increases as $\alpha$ decreases. Therefore, only those collisional quantities that contain low powers of $g$ and $\cos \theta$ (such as $\chi^{(-)}$and $\omega$ ) will be very sensitive to this breakdown as the inelasticity increases, while physical quantities involving higher powers of $g$ and $\cos \theta$, such as the temperature, pressure, or energy dissipation will be well approximated by their molecular chaos counterparts.

\section{Velocity correlations at contact}

In the previous section, we have considered the pair- distribution function $f^{(2)}\left(\mathbf{c}_{1}, \mathbf{c}_{2}, \boldsymbol{\sigma}\right)$ in the precollision state, and have examined how molecular chaos is broken down, and which physical quantities are most sensitive to it. Now we will analyze the effect of the breakdown of molecular chaos on collisional statistics.

We show in Fig. 8 different velocity collisional averages at $\phi=0.05$. In the simulations, these quantities are obtained by averaging over successive collision events in the steady state. We first observe that the simulation results in Fig. 8 approach for $\alpha \rightarrow 1$ the analytic results for elastic spheres, calculated in Eq. (24). At small inelasticities, the simulation data follow the trends of the theoretical prediction with systematic deviations depending on the quantity considered. For instance, the behavior of the center- of-mass motion $\left\langle G^{2}\right\rangle_{\text {coll }}$ is close to the analytical prediction of Eq. (24) in the whole range of $\alpha$ values. This indicates that the center of mass velocity $\mathbf{G}$ is not correlated with the relative velocity $\mathbf{g}$. Consequently, $f^{(2)}\left(\mathbf{c}_{1}, \mathbf{c}_{2}, \boldsymbol{\sigma}\right)$ in the collisional average (11) factorizes, and we may expect the contributions in numerator and denominator in Eq. (11) coming from $\mathbf{G}$ integrations to cancel. Consistent with this behavior, we observe that the two curves in Fig. 8, $\left\langle c_{1}^{2}\right\rangle_{\text {coll }}$ (labeled by circles) and $\left\langle\mathbf{c}_{1} \cdot \mathbf{c}_{2}\right\rangle_{\text {coll }}$ (labeled by squares), are symmetric around $1 / 2$. In Eqs. (A4) and (A5) of the Appendix, these quantities have been expressed in reduced moments

$$
\left\langle\mathbf{c}_{1} \cdot \mathbf{c}_{2}\right\rangle_{\mathrm{coll}}=\frac{1}{2}-\frac{3}{4} \frac{b_{31}}{b_{11}},
$$

$$
\left\langle c_{1}^{2}\right\rangle_{\mathrm{coll}}=\frac{1}{2}+\frac{3}{4} \frac{b_{31}}{b_{11}}
$$

where

$$
b_{n m}=\frac{M_{n m}(T)}{M_{n m}^{\mathrm{E}}(T)}=\left(\frac{T_{\mathrm{E}}}{T}\right)^{n / 2} B_{n m} .
$$

The reduced moments have been measured independently (see Figs. 6 and 7), and used to calculate the expressions (44) and (45). The results have been plotted in Fig. 8 as dashed and dashed-dotted lines, which agree very well with the direct measurements of these quantities as collisional averages, shown in Fig. 8, respectively, as squares and circles. In deriving Eq. (44) and (45), we have again used that the velocity

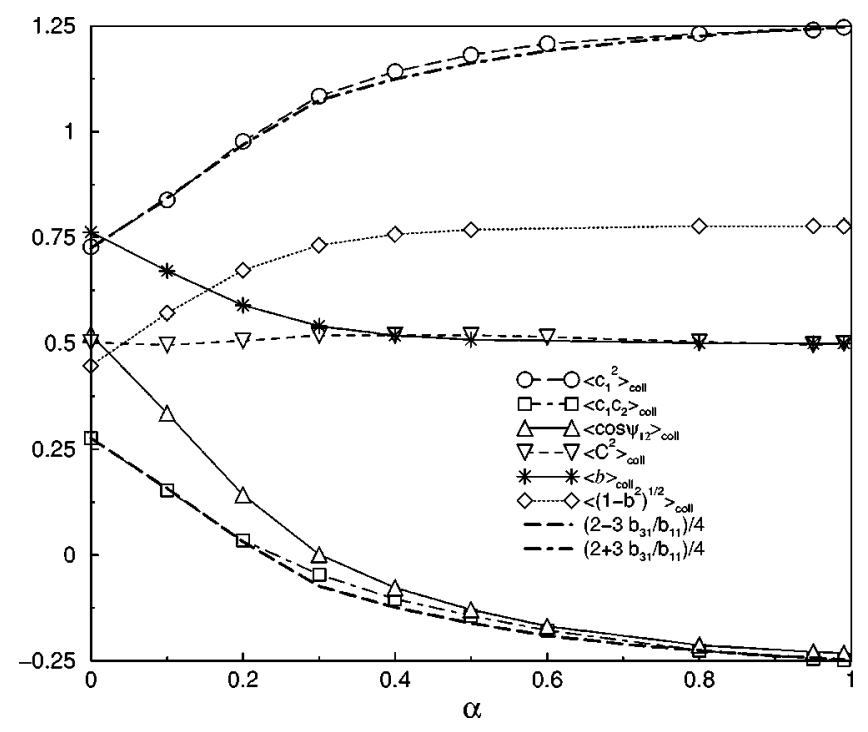

FIG. 8. Values of different collisional averages obtained in MD simulations, as a function of $\alpha$ at $\phi=0.05$ (see Sec. II B for definitions). For $\alpha<0.5$, the random rotation introduced to avoid inelastic collapse has a maximum deviation angle of $2.5^{\circ}$. The symbols $b_{n m}$ are defined in Eq. (45). 


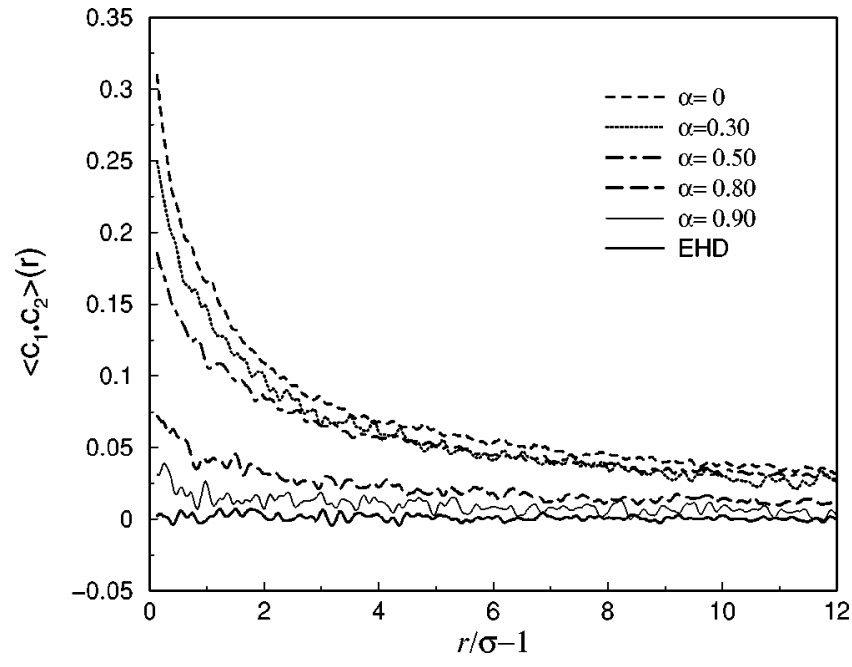

FIG. 9. Distribution of $\left\langle\mathbf{c}_{1} \cdot \mathbf{c}_{2}\right\rangle(r)$ as a function of the distance between the particles at $\phi=0.05$.

variables $\mathbf{G}$ and $\mathbf{g}$ are statistically uncorrelated. The present results strongly support this assumption.

The correlation $\left\langle\hat{\mathbf{c}}_{1} \cdot \hat{\mathbf{c}}_{2}\right\rangle_{\text {coll }}=\left\langle\cos \psi_{12}\right\rangle_{\text {coll }}$, also plotted in Fig. 8, cannot be expressed in $B$ moments. However, the approximate relation already employed to show that $\left\langle\cos \psi_{12}\right\rangle_{\text {coll }} \simeq\left\langle\mathbf{c}_{\mathbf{1}} \cdot \mathbf{c}_{2}\right\rangle_{\text {coll }} /\left\langle c^{2}\right\rangle_{\text {coll }}$ in Sec. II C, holds for the simulation data over the whole range of inelasticities. As the system becomes more inelastic, the typical "temperature" of colliding particles (defined as the collisional average $\left\langle c^{2}\right\rangle_{\text {coll }}$ ) decreases and even becomes lower that the unconstrained average $\left\langle c^{2}\right\rangle$ that defines the temperature. On the other hand, as already noted below $(24),\left\langle c^{2}\right\rangle_{\text {coll }}=5 / 4>1$ in the elastic limit. This decrease of $\left\langle c^{2}\right\rangle_{\text {coll }}$ is directly related to the increase of the small $g$ portion of phase space where molecular chaos is violated. At small $\alpha$, most of the collisions occur between particles with small and even vanishing relative velocities. An extreme example is the inelastic collapse, mentioned in the Introduction.

The correlation function $\left\langle\mathbf{c}_{1} \cdot \mathbf{c}_{2} / g\right\rangle_{\text {coll }}$ for the freely evolving IHD fluid has been simulated by Soto and Mare-schal, and was shown to be small, but nonvanishing [18].

We have also investigated $r-v$ correlations by measuring the expectation value of $\left\langle\mathbf{c}_{1} \cdot \mathbf{c}_{2}\right\rangle(r)$ for two particles separated by a distance $r$, as defined in Eq. (42). The results are shown in Figs. 9 and 10. The plot shows an intermediate range of $r$ values with an exponentially decaying correlation. It is again of interest to compare the extrapolation of the static correlation $\left\langle\mathbf{c}_{1} \cdot \mathbf{c}_{2}\right\rangle(r \rightarrow \sigma)$ with its dynamic counter part $\left\langle\mathbf{c}_{1} \cdot \mathbf{c}_{2}\right\rangle_{\text {dyn }}$ calculated at collision. The results, derived in Eqs. (A8) and (A9) of the Appendix, read for hard disks

$$
\left\langle\mathbf{c}_{1} \cdot \mathbf{c}_{2}\right\rangle_{\mathrm{dyn}}=\frac{1}{2}\left(1-\frac{b_{20}}{b_{00}}\right)+\frac{1-\alpha}{4} \frac{b_{22}}{b_{00}} .
$$

The first term on the right-hand side (RHS) represents the precollision part,

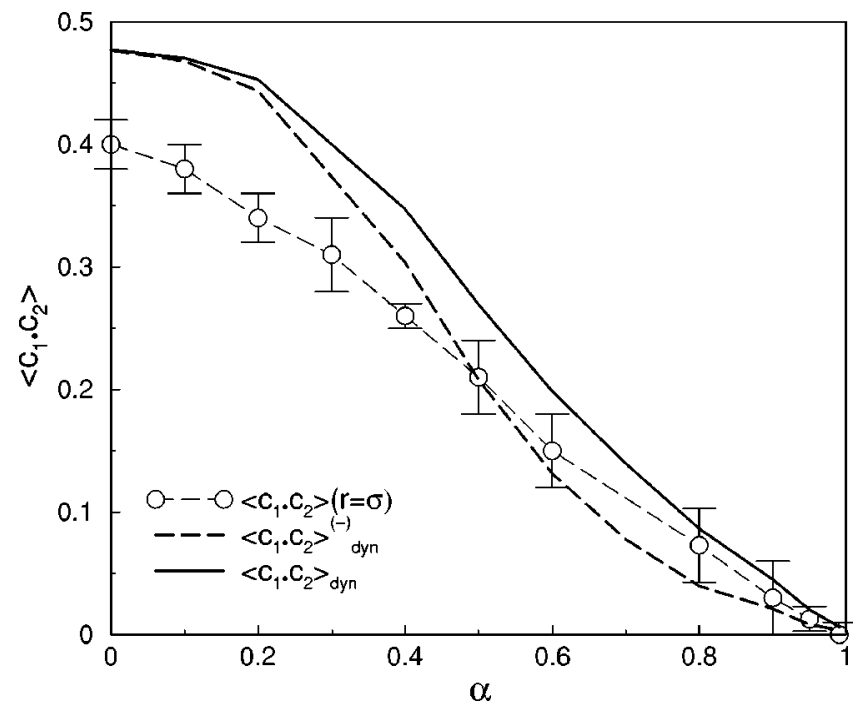

FIG. 10. Mean-velocity-velocity correlation function at contact, as extrapolated from $\left\langle\mathbf{c}_{1} \cdot \mathbf{c}_{2}\right\rangle(r)$ (previous figure), compared with the dynamic analogs $\left\langle\mathbf{c}_{1} \cdot \mathbf{c}_{2}\right\rangle_{\text {dyn }}$ and $\left\langle\mathbf{c}_{1} \cdot \mathbf{c}_{2}\right\rangle_{\text {dyn }}^{(-)}$, defined in Eq. (A8) and (A9).

$$
\left\langle\mathbf{c}_{1} \cdot \mathbf{c}_{2}\right\rangle_{\mathrm{dyn}}^{(-)}=\frac{1}{2}\left(1-\frac{b_{20}}{b_{00}}\right) .
$$

Figure 10 compares the extrapolation $\left\langle\mathbf{c}_{1} \cdot \mathbf{c}_{2}\right\rangle(r \rightarrow \sigma)$ (circles) of the static correlation with its dynamic analogs (46) and (47). The numerical data for both correlations agree well for $\alpha \gtrsim 0.8$, but for $\alpha \lesssim 0.5$, the dynamic correlation (solid line) is substantially larger than the static one. This is consistent with the difference between $\chi^{(-)}$and $Y^{(-)}$observed in Fig. 4. For comparison, the dynamic precollision correlation (dashed line) is also shown. It should be noted that the divergence of $f^{(2)}$ at small $g$ and small $\cos \theta$ implies in particular that $B_{00} \gg B_{20}>B_{22}$, so that Eq. (46) predicts that the dynamic correlation at contact $\left\langle\mathbf{c}_{1} \cdot \mathbf{c}_{2}\right\rangle_{\text {dyn }}$ should increase at $\alpha \rightarrow 0$ and saturate close to $1 / 2$. By the same arguments, its precollision part in Eq. (47) approaches the same limit. This can be observed in Fig. 10.

The velocity correlation $\left\langle\mathbf{c}_{1} \cdot \mathbf{c}_{2}\right\rangle_{\text {coll }}$ in Eq. (44) involves the reduced moments $b_{31}$ and $b_{11}$. Consistent with the scenario, developed in Sec. III $C$, the divergence of $f^{(2)}\left(\mathbf{c}_{1}, \mathbf{c}_{2}, \boldsymbol{\sigma}\right)$ near $g=0$ and $\cos \theta=0$ is largely suppressed in these higher moments, which remain finite for $\alpha \rightarrow 0$, where $b_{11} \simeq 4 b_{31}$. Consequently, $\left\langle\mathbf{c}_{1} \cdot \mathbf{c}_{2}\right\rangle_{\text {coll }}$ does not approach the value $1 / 2$ as $\alpha \rightarrow 0$, but a value close to 0.3 , as can be deduced from Fig. 8 .

\section{E. Grazing collisions}

The data in Fig. 8 for $\left\langle\mathbf{c}_{1} \cdot \mathbf{c}_{2}\right\rangle_{\text {coll }},\left\langle\cos \psi_{12}\right\rangle_{\text {coll }}$, $\left\langle\sqrt{1-b^{2}}\right\rangle_{\text {coll }}$, and $\langle b\rangle_{\text {coll }}$ clearly illustrate that the violation of molecular chaos strongly increases with increasing inelasticity. Consider first the average

$$
\langle b\rangle_{\mathrm{coll}}=\int_{0}^{1} \mathrm{~d} b b P(b) .
$$




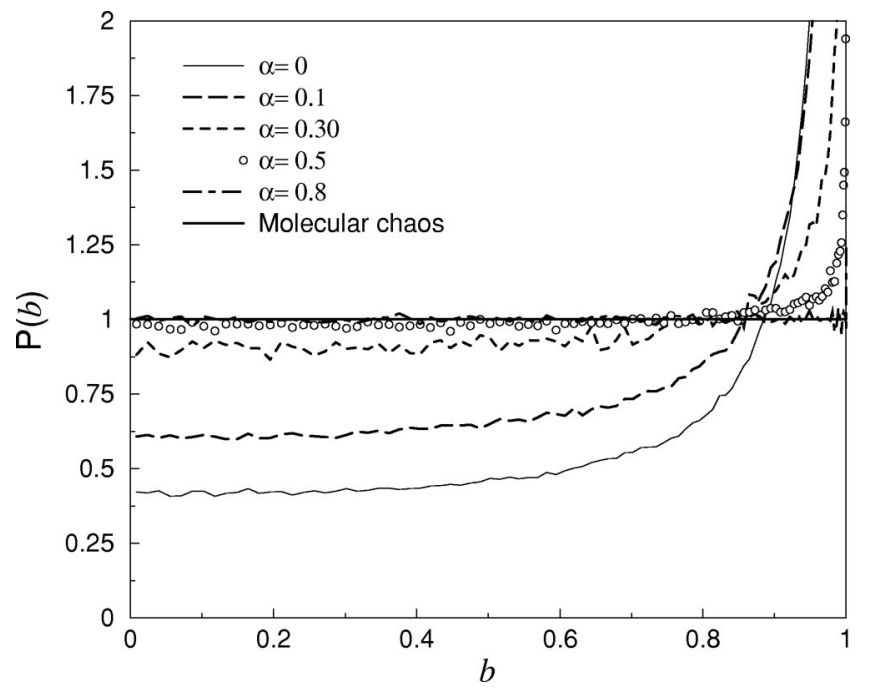

FIG. 11. Distribution of the impact parameter $b$ for different $\alpha$ values for $\phi=0.05$.

This average remains at a plateau value $1 / 2$ for $\alpha \gtrsim 0.5$, which is determined by the uniform distribution $P(b)$ corresponding to molecular chaos in two dimensions. Recall that the value $1 / 2$ holds regardless of the functional form of the velocity distribution function $f$. It is thus a good probe for molecular chaos breakdown. Moreover, from its trend we can also estimate the way in which such a breakdown takes place. Specifically, as the inelasticity increases the average value increases by about $50 \%$, which indicates a strong bias toward grazing collisions. To illustrate this, we model the normalized distribution of impact parameters as a uniform background and a "half" delta peak at $b=1$, i.e., $P(b)=1$ $-p+2 p \delta(1-b)$, where $p$ is the fraction of grazing collisions. This yields the average $\langle b\rangle_{\text {coll }}=1 / 2(1+p)$, which implies, according to Fig. 8, that at $\alpha=0.0,0.1$, and 0.3 , respectively, a fraction of 50,35, and $5 \%$ is grazing at $\phi=0.05$. This qualitative picture is supported in a more quantitative manner in Fig. 11, which shows the measured $P(b)$, which is strongly peaked near grazing collisions $(b=1)$. At small inelasticity, all impact parameters are equally probable as expected on the basis of molecular chaos, and consistent with Fig. 8. Only for $\alpha \lesssim 0.5$ deviations become significant: upon decreasing the coefficient of restitution, collisions with a larger impact parameter occur more frequently, implying an increase of the frequency of grazing collisions. The behavior of $P(b)$ is then fully consistent with the divergence of $f^{(2)}$ at small $\cos \theta$, discussed in Sec. III C.

To avoid inelastic collapse for $\alpha \leqslant 0.5$, the postcollision velocities of colliding pairs are rotated over a small random angle as described in Refs. [36,10], with the important restriction mentioned at the beginning of Sec. III. Alternative algorithms to avoid inelastic collapse are described in Ref. [37]. For $\alpha>0.5$, no such rotation was applied. To check if the deviations of the impact parameter for $\alpha \lesssim 0.5$ are due to this applied rotation, we have also performed simulations where even for $\alpha>0.5$ a random rotation was applied. Regardless of the applied random rotation, we found $\langle b\rangle_{\text {coll }}$ close to $1 / 2$ for $\alpha \gtrsim 0.5$. Both Figs. 8 and 11 show that for

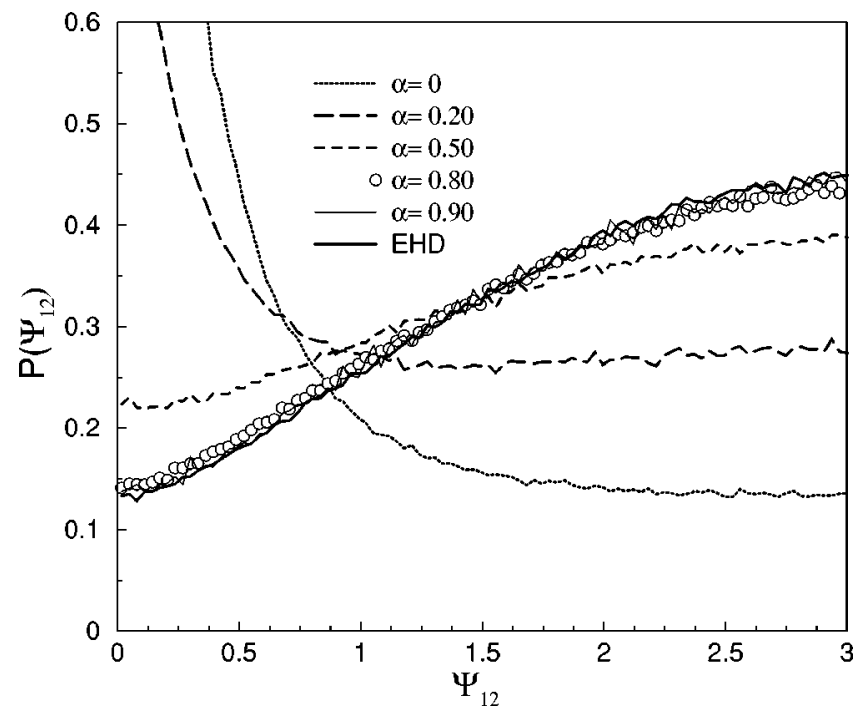

FIG. 12. Distribution of the relative orientation of the velocities at collision $\left(\cos \psi_{12} \equiv \hat{\mathbf{c}}_{1} \cdot \hat{\mathbf{c}}_{2}\right)$ at a packing fraction of $5 \%$.

$\alpha \lesssim 0.5$ molecular chaos is strongly violated, and that the violation is weaker in the small inelasticity regime. The average $\left\langle\sqrt{1-b^{2}}\right\rangle_{\text {coll }}$ supports the same conclusions.

The data for $\left\langle\mathbf{c}_{1} \cdot \mathbf{c}_{2}\right\rangle_{\text {coll }}$ and $\left\langle\cos \psi_{12}\right\rangle_{\text {coll }}$ in Fig. 8 are consistent with the predominance of grazing collisions at large inelasticities. They show the average relative angle between the velocities of the incoming particles, which has a strong $\alpha$ dependence and no plateau value near the elastic limit. Near $\alpha=1$, the particles are on average on approaching trajectories with $\left\langle\cos \psi_{12}\right\rangle_{\mathrm{coll}} \simeq-0.25$ and $\left\langle\psi_{12}\right\rangle_{\mathrm{coll}} \simeq 105^{\circ}$. As $\alpha$ decreases, $\left\langle\cos \psi_{12}\right\rangle_{\text {coll }}$ increases linearly to a value 0.50 , while $\left\langle\psi_{12}\right\rangle_{\text {coll }}$ approaches $60^{\circ}$, at $\alpha=0$. This corresponds to collisions of more or less parallel-moving pairs of particles, where faster particles overtake slower ones.

Figures 12 and 13 show the distribution of relative orientations of incoming velocities. The distribution of angles between the incoming particles $\left(\psi_{12}\right)$ shows moderate devia-

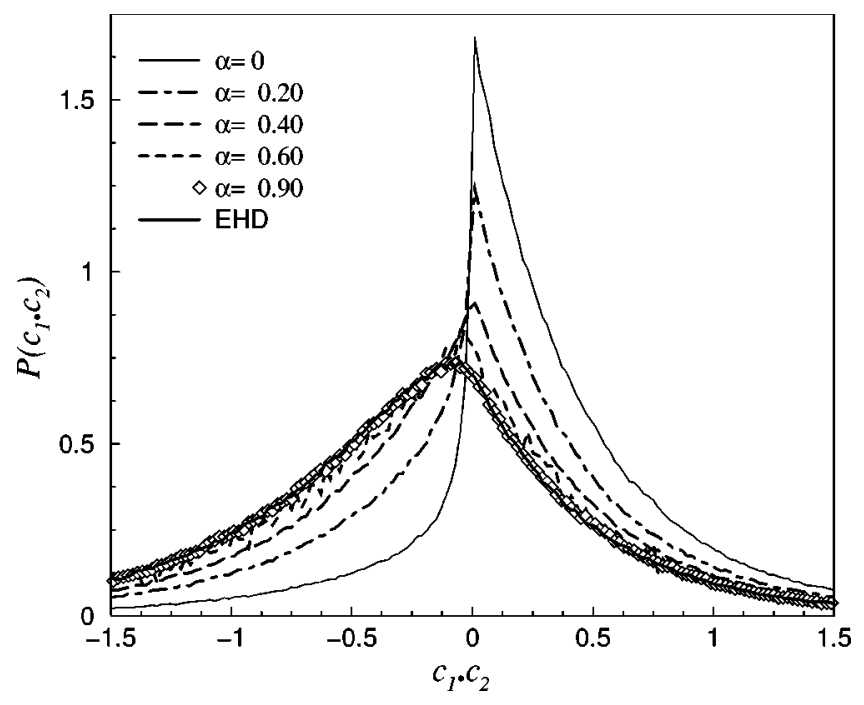

FIG. 13. Distribution of relative velocities $\mathbf{c}_{1} \cdot \mathbf{c}_{2}$ of colliding inelastic disks at $\phi=0.05$. 
TABLE I. Frequency of recollision events as a function of the inelasticity (see text for the definition of $\mathcal{R}_{0}$ and $\mathcal{R}_{1}$ ). The packing fraction is $\phi=0.2$ and the system contains $N=5000$ disks.

\begin{tabular}{lcc}
\hline \hline$\alpha$ & $\mathcal{R}_{0}$ & $\mathcal{R}_{1}$ \\
\hline 0 & $52 \%$ & $18 \%$ \\
0.4 & $14 \%$ & $15 \%$ \\
0.6 & $4 \%$ & $15 \%$ \\
0.95 & $0.15 \%$ & $7 \%$ \\
1.0 & $0 \%$ & $6.7 \%$ \\
\hline \hline
\end{tabular}

tions from what is expected for an elastic system in the range $0.5 \leq \alpha<1$. As an analytic expression for elastic disks is not available, deviations are compared with the simulation results for elastic hard disks (in the absence of a random external force). At $\alpha=0.5$, the frequency of collisions of parallel-moving particles is strongly increased, a trend that is enhanced upon increasing the inelasticity. Finally, the probability distribution $P\left(\mathbf{c}_{1} \cdot \mathbf{c}_{2}\right)$ is shown in Fig. 13. When the inelasticity increases, this distribution becomes more peaked around the origin, as the colliding particles on average move more slowly relative to each other. In the mean time, the typical angle $\psi_{12}$ decreases, which causes this peak to shift to positive values.

\section{F. Particle- and noise-induced recollisions}

The mechanism for the breakdown of molecular chaos in classical fluids with conservative interactions are sequences of correlated ring collisions, as discussed in the introduction. The most simple ring collisions are the recollisions (1-2) (1-3) (1-2) and cyclic collisions $(1-2)(2-3)(3-1)$ or permutations thereof [20].

There is strong evidence that the effects of ring collisions are considerably enhanced in fluids with dissipative interactions, such as granular flows, where relative kinetic energy is lost in binary collisions. As a result, the postcollision velocities $\left\{\mathbf{v}_{1}^{*}, \mathbf{v}_{2}^{*}\right\}$ will be on average more parallel than the precollision ones $\left\{\mathbf{v}_{1}, \mathbf{v}_{2}\right\}$ [24], i.e., the trajectories are less diverging than in the elastic case, and there is a much larger $\left\{\mathbf{r}_{3}, \mathbf{v}_{3}\right\}$ phase space, in which particle three will knock, say, particle one back to recollide with particle two.

This increase of phase space is confirmed by gathering recollision statistics. We have counted the fraction of recollisions as a function of $\alpha$, as shown in Table I. The column labeled $\mathcal{R}_{1}$ (recollisions between two partners mediated by a third particle) shows that at a packing fraction $\phi=0.2$ in the elastic case $(\alpha=1)$ only a fraction of $6.7 \%$ of all collisions is a recollision. This frequency gradually increases to about $15 \%$ at $\alpha=0.4$.

In the randomly driven IHS fluid, there is the additional effect of noise-induced recollisions that do not require the intervention of other particles. This type of recollision (denoted $\mathcal{R}_{0}$ ) occurs with high probability when the relative velocity after collision is so small that it may be simply reversed by a random kick. At $\alpha=0.6$, the frequency of noise-induced recollisions is about $4 \%$, and it increases to $52 \%$ at $\alpha=0$ (see column $\mathcal{R}_{0}$ in Table I). The effect is of importance at all densities, because it does not require the mediation of a third particle. Indeed, at a low-packing fraction of $1 \%$ and in the completely inelastic case $\alpha=0$, the frequency of $\mathcal{R}_{0}$-like events is still $34 \%$, while $\mathcal{R}_{1}$-like events have dropped to $5 \%$. Moreover, we have verified that inclusion of rotation-induced recollisions modifies most of the collisional quantities we have analyzed, increasing their deviations with respect to the molecular chaos prediction.

At present, more quantitative theories or estimates of the effect of both types of recollisions and other ring collisions on the short-range behavior of the pair-distribution function $f^{(2)}\left(x_{1}, x_{2}\right)$ are lacking. A natural way to incorporate the noise-induced recollisions into a kinetic theory description would be to include them into an effective two-particle scattering operator, which transforms an asymptotic precollision state of two independent particles into an asymptotic postcollision state, without involving intermediate two-particle scattering states, as in the present case. This may lead to an instantaneous Boltzmann collision term (without memory effects), provided the mean free time and the time between random kicks are very well-separated (dilute gases). Such a description would suppress the recollions of type $\mathcal{R}_{0}$, and make the violation of molecular chaos less severe, say comparable to the freely evolving IHD fluid.

\section{G. Cold dense inhomogeneities}

In Ref. [10], we have shown by analyzing the Fourier modes of the granular hydrodynamic equations, which are valid for small inelasticities (say $\alpha>0.7$ ), that the NESS in a randomly driven IHS fluid is linearly stable against spatial inhomogeneities. Consequently, when observed over sufficiently long times, the NESS should be spatially homogeneous. However, it was also shown that the NESS exhibits strong fluctuations, resulting in long-range spatial correlations in density, flow field, and granular temperature. The observation of density inhomogeneities for large inelasticities has already been reported by Peng and Ohta [8]. These density inhomogeneities, as shown by the snapshot of the density in Fig. 14, are not quasistatic, as in the freely evolving case $[38,36,39,24]$, but seem to behave as dynamic assemblies of particles that dissolve and reassemble again. Also, for a uniform shear flow, dynamical density inhomogeneities have been reported [40]. The existence of density inhomogeneities was already suggested by the static pairdistribution functions $g(r)$, which showed an enhancement of the first few maxima as compared to their elastic values (see Fig. 3).

In Fig. 8, we show that the mean energy $\left\langle c_{1}^{2}\right\rangle_{\text {coll }}$, of particles aiming to collide, is above the mean, $\left\langle c^{2}\right\rangle=1$, for small inelasticity. It decreases from its elastic value $5\left\langle c^{2}\right\rangle / 4$ with decreasing $\alpha$, then crosses the mean value value $\left\langle c^{2}\right\rangle$ $=1$ at $\alpha \simeq 0.2$, and further decreases to approximately $0.7\left\langle c^{2}\right\rangle$ at $\alpha=0$.

It is interesting to observe that in the strong dissipation range, the mean kinetic energy or granular temperature of particles that are about to collide is lower than the average temperature. We combine this observation with Figs. 3(a) and 3(b) of Peng and Ohta [8], which show that essentially 


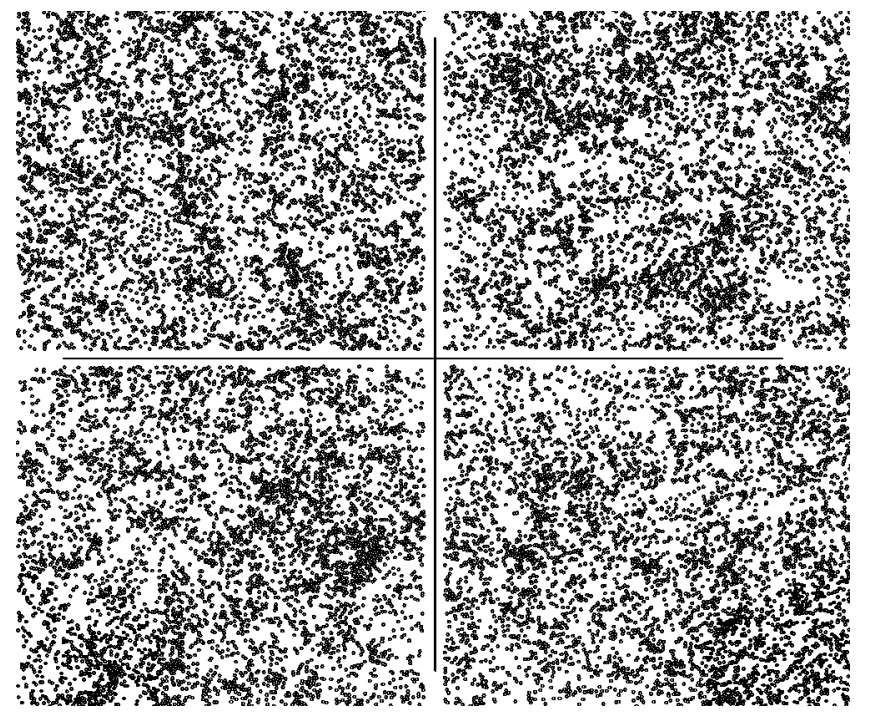

FIG. 14. To illustrate the slow reorganization of density inhomogeneities, four consecutive snapshots of the system are shown at $\alpha=0.1, \phi=0.2$, and $N=5000$ (the full simulation box is displayed). The time interval between two consecutive snapshots corresponds to 50 collisions per particle.

all collisions occur inside "cold" regions of high densities. This last observation applies even more so to undriven IHS fluids $[38,41]$. We expect that, also in the randomly driven IHS fluid, the majority of collisions takes place inside cold high-density regions.

If the predominance of cold particles in strongly inelastic collisions, $\left\langle c^{2}\right\rangle_{\text {coll }}\left\langle\left\langle c^{2}\right\rangle\right.$ is indeed a signal for the appearance of density inhomogeneities, then Fig. 8 suggests that at a packing fraction $\phi=0.05$ density inhomogeneities may occur for $\alpha \lesssim 0.2$. This is indeed confirmed by the snapshots in Figs. 14. In Fig. 15, we illustrate the existence of cold inhomogeneous dense regions for $\alpha=0.2$ and $\phi=0.2$. The particles with a less (more) than median kinetic energy are shown on the left (right). The formation of inhomogeneities is more clear for the colder particles. The temporal evolution of these regions show that they dissolve after some time, while inhomogeneous regions appear. The formation of "living" inhomogeneous regions may be understood using the hydrodynamic picture put forward in [10], where it was shown that the structure factor behaves as $S(\mathbf{k}) \sim k^{-2}$, implying density correlations increasing with distance as $\ln (r)$ in two dimensions. These long range spatial correlations induce a slowing down of the dynamics, as in critical phenomena. This, in turn, implies the slow decay of density perturbations, which could lead to visible density inhomogeneities as the kicking frequency is reduced (in this respect, see Refs. [6]). We may also expect that upon decreasing the forcing frequency, the dynamics should be closer to its "free cooling" counterpart so that well-defined clusters are then likely to appear.

More details about the predominance of cold particles, among those involved in collisions, may be seen in Fig. 16, which shows the constrained probability distribution $P(c)$, defined in Sec. II C and obtained from MD-simulations at different inelasticities. For $\alpha \lesssim 0.5$, the distribution has significantly shifted to smaller impact velocities. For the completely inelastic case, collision events involving "immobile" particles are more than twice as frequent as for the elastic case. The second moment of the distribution displayed in Fig. 16 decreases when increasing the inelasticity. In fact, all
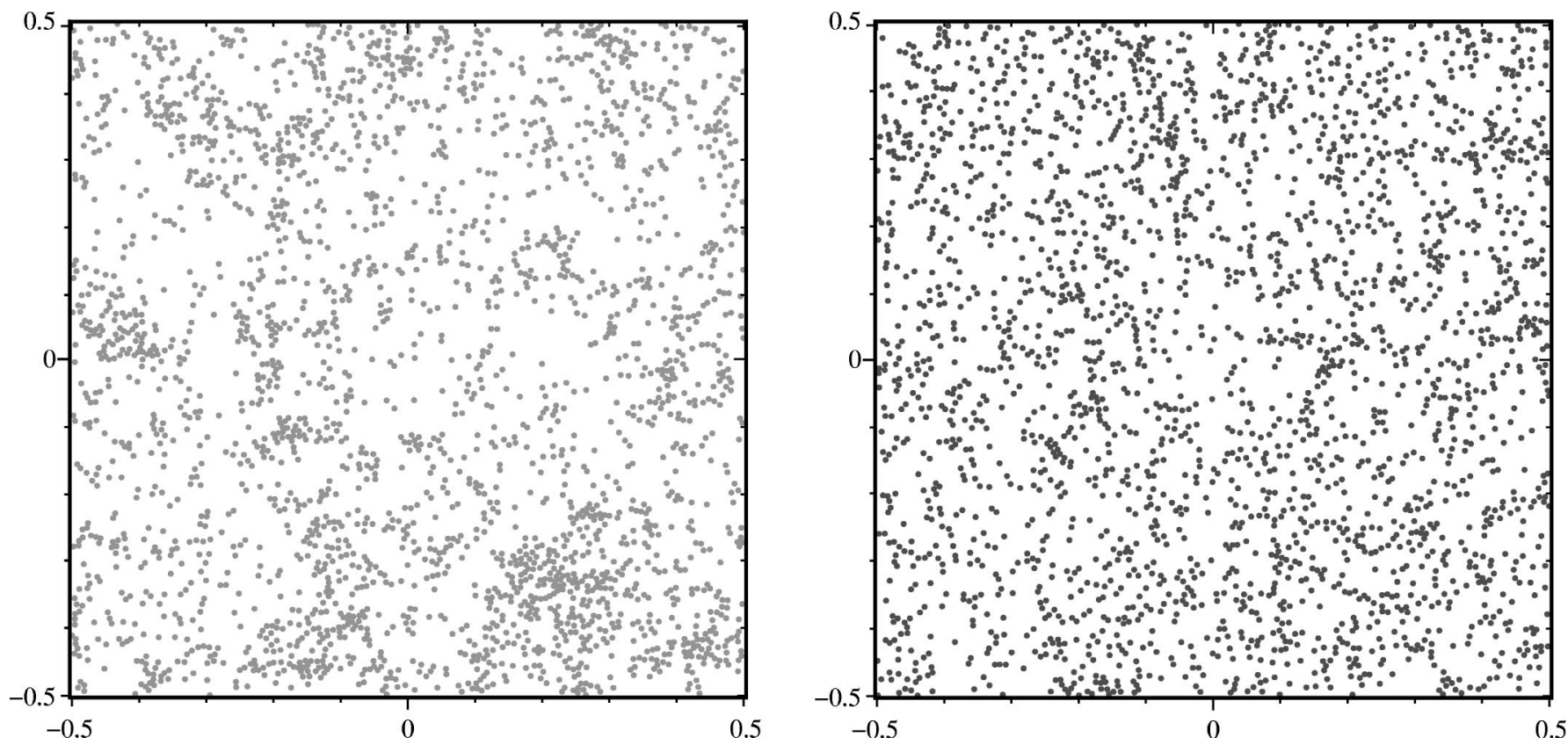

FIG. 15. Snapshot of a typical instantaneous configuration of the system at $\alpha=0.2, \phi=0.2$, and $N=5000$. To illustrate the existence of cold dense inhomogeneities, on the left (right) the particles with a less (more) than median kinetic-energy $\mathcal{E}^{*}$ are shown at real scale (i.e., the cutoff $\mathcal{E}^{*}$ is chosen such that there are exactly half of the particles on each graph). Lengths on the $x$ and $y$ axes are expressed in units of the simulation box length. 

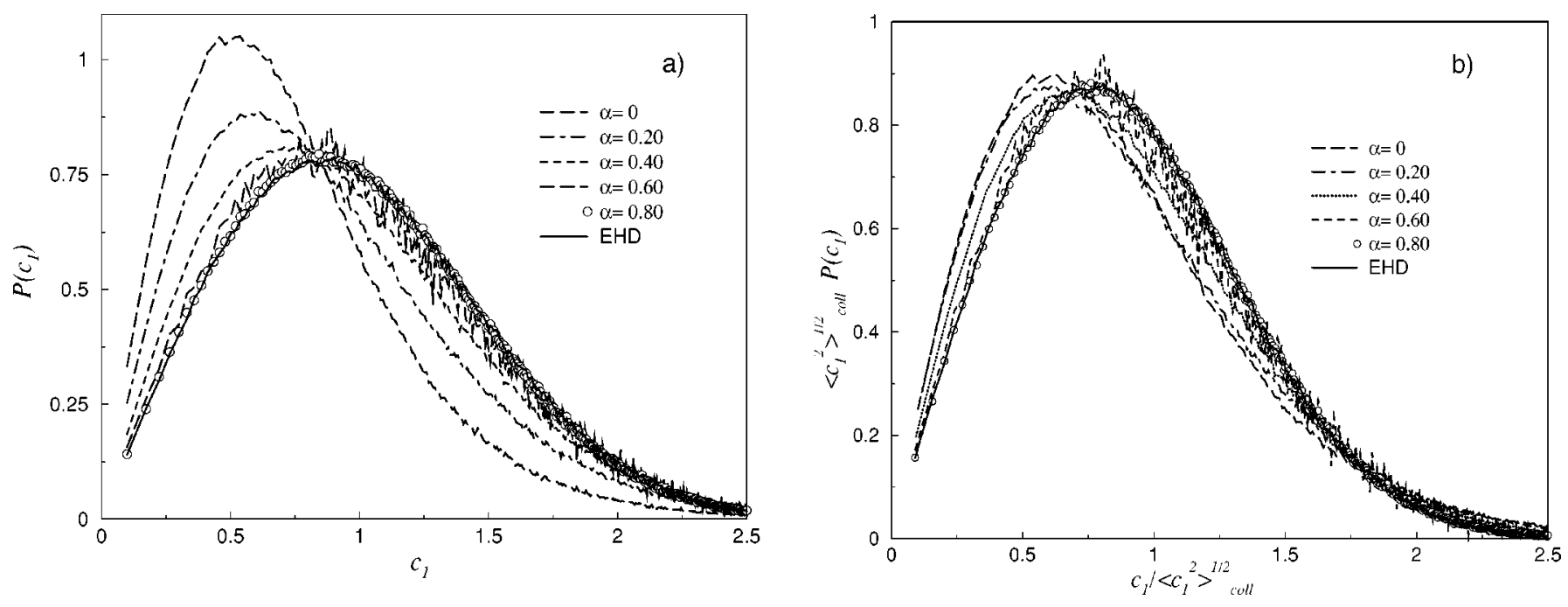

FIG. 16. Velocity distribution of the colliding particles at $\phi=0.05$ and $N=5041$ : (a) Original distribution; (b) scaled velocity distributions as a function of the rescaled velocity $c / \sqrt{T(\alpha)}$ for different values of $\alpha$.

functional forms with simulation data at different $\alpha$ can essentially be collapsed onto a single universal curve (the elastic one) by plotting $\sqrt{T(\alpha)} P(c \mid \alpha)$ as a function of $c / \sqrt{T(\alpha)}$, where $T(\alpha)=\left\langle c^{2}\right\rangle_{\text {coll }}$ is the mean temperature of a particle at collision. The collapse plot is shown in Fig. 16(b). This data collapse confirms the concept of cold dense regions dominating the energy dissipation. This could point to a possibly relevant two fluid picture of a "hot" dilute background gas coexisting with continuously rearranging configurations of "cold" dense regions.

\section{CONCLUSION}

We have performed extensive MD simulations to study the kinetic properties and short-range correlations in the nonequilibrium steady state of a randomly driven fluid of inelastic hard disks, as a model for fluidized granular material. The MD results have been compared with kinetic theory predictions derived from the Enskog-Boltzmann equation, properly modified with a Fokker-Planck diffusion term $\xi_{0}^{2}(\partial / \partial \mathbf{v})^{2}$ to account for the applied random driving force [9].

It appears that the kinetic theory predictions, based on molecular chaos, are essentially in agreement with the MD results for small inelasticities $(\alpha \gtrsim 0.5)$ at $\phi=0.05$. For larger inelasticities, the deviations from the molecular chaos predictions start to become manifest: the radial distribution function at contact differs strongly from its local equilibrium form; there is a predominance of grazing collisions. When increasing $\phi$, the effects of the inelastic collisions become relevant at smaller inelasticities; e.g., at $\phi=0.2$ and $\phi=0.5$, we observe already significant deviations for $\alpha \leqslant 0.7$.

To avoid inelastic collapse of the system at low $\alpha$, we have implemented a modified rotation procedure (see the beginning of Sec. III). In its original version, this procedure induces dramatic violations of molecular chaos. It could then be argued that the important deviations of low-order-reduced moments $B_{n m}$ are also spurious consequences of the above rotation procedure. However, we checked that circumventing the collapse by applying elastic collisions when the relative velocity of a pair is below a certain cutoff [37], also induces very important violations of molecular chaos (quantified by $B_{00}$ for instance), unless the cutoff is chosen unphysically high.

Sequences of ring collision processes, which lead to the breakdown of molecular chaos in classical fluids with conservative interactions, are strongly enhanced in fluids with dissipative interactions, such as rapid granular flows. We have analyzed how molecular chaos is broken, i.e., essentially only through pairs of colliding particles at very small relative velocities. This means that molecular chaos is violated only in a small portion of phase space, implying that only certain physical properties will be sensitive to this violation. This explains why quantities such as the collision frequency, or the pair-distribution function at contact are very sensitive to the inelasticity parameters, while others such as the pressure or the energy dissipation rate are well approximated by their Enskog prediction. Disentangling the effects of hard disk and noise-induced correlations remains an interesting point to explore. The studies performed in a freely evolving IHS fluid also shows the predominance of grazing collisions at long times. The fact that we have observed an analogous behavior for this homogeneous steady state indicates that the mechanism of breakdown of molecular chaos in granular fluids through grazing collisions is generic for this type of fluids.

The extra feature of noise-induced recollisions, which do not require mediation of a third particle, will further enhance the violation of the molecular chaos assumption. A natural way to develop a kinetic theory for randomly driven fluids, thereby presumably restoring the validity of the molecular chaos assumption in the dilute gas case, could be to include the noise-induced recollisions in an effective two-particle scattering operator. It would be of interest to study its properties, either analytically or by simulating a two-particle inelastic collision in the presence of external noise. An additional theoretical complication here is the validity of the Boltzmann Eq. (1) with Fokker-Planck diffusion term due to the fact that there are two limits involved when dealing with 
hard spheres in combination with external white noise. The actual properties of the effective collision operator depend on the order in which both limits are taken. In the simulations, one always takes the hard sphere limit first, while the white noise is approximated by discrete kicks that are applied to the particles at discrete times.

In Ref. [10], we have calculated the equal-time spatial correlations of the fluctuations in the hydrodynamic densities in the NESS. Here, we have focused on the dynamic properties of these enhanced fluctuations, in particular of the dynamic inhomogeneities observed in the density field. The collisional velocity moments, introduced in Sec. II and measured in MD simulations, reveal that the dense regions consist mostly of particles colder than average. This is clearly shown in the velocity distribution $P(v \mid \alpha)$ of particles that are about to collide.

The MD simulations have been carried out in the limit in which the time interval between the external random kicks is much shorter than the mean free time between collisions. In this limit, regions with density larger than average are not seen to survive for a long time. Rather, they form, dissolve, and reappear elsewhere. The spatial correlations analyzed in [10] show long-range correlations, which imply also a slowdown in the temporal decay of density perturbations. Therefore, we expect than the decrease of the kicking frequency will be accompanied by the appearance of apparent clusters. This fact, together with the shape modification of the velocity distribution $P(v \mid \alpha)$ (see Fig. 16) suggests the picture of a two-fluid model, in which a "hotter" more dilute background gas coexists with continuously rearranging configurations of "cold" dense clusters. This point remains open for subsequent investigation; for example, it would be interesting to analyze separately the collisional statistics in the dense and dilute regions to assess the role of density fluctuations.

\section{ACKNOWLEDGMENTS}

E.T. and I.P. thank D. Frenkel and B. Mulder for the hospitality of their groups at AMOLF. I.P thanks M.E. Cates for his hospitality in the University of Edinburgh, where part of this work was done. We acknowledge stimulating discussions with H.J. Herrmann, S. Luding, R. Soto, M. Mareschal, and J. Piasecki. T.v.N. and I.P. acknowledge support of the foundation "Fundamenteel Onderzoek der Materie (FOM)," which is financially supported by the Dutch National Science Foundation (NWO).

\section{APPENDIX: REDUCED MOMENTS $B_{n m}(T)$}

In the body of the paper, we have considered the collisional averages $\left\langle g^{n}|\cos \theta|^{m}\right\rangle_{\text {coll }}$ and the moments $M_{n, m}(T)$ and $B_{n, m}(T)$. We first list the Enskog values of these quantities, which have have been calculated from its definitions, given below Eq. (28): i.e.,

$$
M_{n m}^{\mathrm{E}}\left(T_{\mathrm{E}}\right)=v_{\mathrm{E}}^{n} \chi_{\mathrm{E}} 2^{n / 2} \frac{\Gamma((d+n) / 2) \Gamma((m+1) / 2)}{\sqrt{\pi} \Gamma((d+m) / 2)},
$$

$$
\left\langle g^{n}|\cos \theta|^{m}\right\rangle_{\mathrm{coll}}^{\mathrm{E}}=2^{n / 2} \frac{\Gamma((d+n+1) / 2) \Gamma((m+2) / 2)}{\Gamma((d+m+1) / 2)} .
$$

Many physical quantities of interest may be expressed in terms of reduced moments $B_{n m}$, as already illustrated in Sec. II C for $\chi^{(-)}, \omega, p$, and $\Gamma$. Analogous relations hold for the velocity moments $\left\langle g^{n}\right\rangle_{\text {coll }}$, which are proportional to $M_{n+1,1}$. This yields

$$
\left.B_{n+1,1}=\frac{\omega}{\omega_{\mathrm{E}}} \times \frac{\left\langle v_{12}^{n}\right\rangle_{\text {coll }}}{\left\langle v_{12}^{n}\right\rangle_{\text {coll }}^{\mathrm{E}}}=\frac{\omega}{\omega_{\mathrm{E}}} \times \frac{\left\langle g^{n}\right\rangle_{\text {coll }}}{\left\langle g^{n}\right\rangle_{\text {coll }}^{\mathrm{E}}} \frac{T}{T_{\mathrm{E}}}\right)^{n / 2},
$$

where the denominator has been calculated in Eq. (A2).

Velocity correlations between nearby particles may also be expressed in the reduced moments $B_{n m}(T)$. First, consider the constrained averages $\left\langle\mathbf{c}_{1} \cdot \mathbf{c}_{2}\right\rangle_{\text {coll }}$, defined in Eq. (24). They contain $\left\langle G^{2}\right\rangle_{\text {coll }}$, which equals d/4 from the MD simulations, in agreement with Eq. (24) (see Fig. 8 of Sec. III). The center of mass velocity $\mathbf{G}$ is consequently uncorrelated with the relative velocity, and independent of the inelasticity. Substitution of $\left\langle G^{2}\right\rangle_{\text {coll }}=d / 4$ in Eq. (24) yields

$$
\begin{gathered}
\left\langle\mathbf{c}_{1} \cdot \mathbf{c}_{2}\right\rangle_{\mathrm{coll}}=\frac{d}{4}-\frac{1}{4}\left\langle g^{2}\right\rangle_{\mathrm{coll}}=\frac{d}{4}-\frac{d+1}{4}\left(\frac{T_{\mathrm{E}}}{T}\right) \frac{B_{31}}{B_{11}}, \\
\left\langle c_{1}^{2}\right\rangle_{\mathrm{coll}}=\frac{d}{4}+\frac{1}{4}\left\langle g^{2}\right\rangle_{\mathrm{coll}}=\frac{d}{4}+\frac{d+1}{4}\left(\frac{T_{\mathrm{E}}}{T}\right) \frac{B_{31}}{B_{11}} .
\end{gathered}
$$

Similarly we find

$$
\begin{aligned}
\left\langle\mathbf{c}_{1} \cdot \mathbf{c}_{2} / g\right\rangle_{\text {coll }}= & \left\langle G^{2} / g\right\rangle_{\text {coll }}-\frac{1}{4}\langle g\rangle_{\text {coll }} \\
= & \frac{d}{4 \sqrt{2}} \frac{\Gamma(d / 2)}{\Gamma((d+1) / 2)} \sqrt{\frac{T}{T_{\mathrm{E}}}} \\
& \times \frac{B_{01}}{B_{11}}\left\{1-\left(\frac{T_{\mathrm{E}}}{T}\right) \frac{B_{21}}{B_{01}}\right\}
\end{aligned}
$$

and

$$
\begin{aligned}
\left\langle\mathbf{c}_{1} \cdot \mathbf{c}_{2} /|g \cos \theta|\right\rangle_{\mathrm{coll}} & =\left\langle G^{2} /|g \cos \theta|\right\rangle_{\mathrm{coll}}-\frac{1}{4}\langle g /|\cos \theta|\rangle_{\mathrm{coll}} \\
& =\frac{d}{4} \sqrt{\frac{\pi}{2}} \sqrt{\frac{T}{T_{\mathrm{E}}}} \times \frac{B_{00}}{B_{11}}\left\{1-\left(\frac{T_{\mathrm{E}}}{T}\right) \frac{B_{21}}{B_{01}}\right\} .
\end{aligned}
$$

Note that the last two averages are vanishing in the elastic case.

In the body of the paper we have considered the extrapolation of the static correlation $\left\langle\mathbf{c}_{1} \cdot \mathbf{c}_{2}\right\rangle(r \rightarrow \sigma)$. Here, we calculate its dynamic analog $\left\langle\mathbf{c}_{1} \cdot \mathbf{c}_{2}\right\rangle_{\text {dyn }}$, obtained by interchanging limits and replacing $f^{(2)}\left(\mathbf{c}_{1}, \mathbf{c}_{2}, r\right)$ under the integral sign in Eq. (41) by its value at contact, $f^{(2)}\left(\mathbf{c}_{1}, \mathbf{c}_{2}, \boldsymbol{\sigma}\right)$. We proceed in the same fashion as in Eqs. (36)-(39), and 
split the numerator in Eq. (42) in a pre- and postcollision part, as done in Sec. II D. One finds after a lengthy calculation,

$$
\left\langle\mathbf{c}_{1} \cdot \mathbf{c}_{2}\right\rangle_{\mathrm{dyn}}=\frac{d}{4}\left\{1-\left(\frac{T_{\mathrm{E}}}{T}\right) \frac{B_{20}}{B_{00}}\right\}+\frac{1-\alpha}{4}\left(\frac{T_{\mathrm{E}}}{T}\right) \frac{B_{22}}{B_{00}} .
$$

Here, the first term on the RHS is its precollision part, i.e.,

$$
\begin{aligned}
\left\langle\mathbf{c}_{1} \cdot \mathbf{c}_{2}\right\rangle_{\mathrm{dyn}}^{(-)} & =\left\langle\mathbf{c}_{1} \cdot \mathbf{c}_{2}|g \cos \theta|^{-1}\right\rangle_{\mathrm{coll}} /\left\langle|g \cos \theta|^{-1}\right\rangle_{\mathrm{coll}} \\
& =\frac{d}{4}\left\{1-\left(\frac{T_{\mathrm{E}}}{T}\right) \frac{B_{20}}{B_{00}}\right\}
\end{aligned}
$$

In Sec. III, these quantities are compared with MD simulations.
[1] F. Melo, P.B. Umbanhowar, and H.L. Swinney, Phys. Rev. Lett. 75, 3838 (1995); P.B. Umbanhowar, F. Melo, and H.L. Swinney, Nature (London) 382, 793 (1996); C. Bizon and H.L. Swinney, in Models and Kinetic Methods for Nonequilibrium Many Body Systems, Vol. 371 of NATO Advanced Studies Series, edited by J. Karkheck (Kluwer, Dordrecht, 2000).

[2] J.S. Olafsen and J.S. Urbach, Phys. Rev. Lett. 81, 4369 (1998); J.S. Urbach, in Granular Gases, Lecture Notes in Physics Vol. 564, edited by T. Pöschel and S. Luding (Springer Verlag, Berlin, 2001).

[3] W. Losert, D.G.W. Cooper, J. Delour, A. Kudrolli, and J.P. Gollub, Chaos 9, 682 (1999).

[4] F. Rouyer and N. Menon, Phys. Rev. Lett. 85, 3676 (2000).

[5] D.R.M. Williams and F.C. MacKintosh, Phys. Rev. E 54, R9 (1996).

[6] A. Puglisi, V. Loreto, U.M.B. Marconi, A. Petri, and A. Vulpiani, Phys. Rev. Lett. 81, 3848 (1998); Phys. Rev. E 59, 5582 (1999).

[7] R. Cafiero, S. Luding, and H.J. Herrmann, Phys. Rev. Lett. 84, 6014 (2000).

[8] G. Peng and T. Ohta, Phys. Rev. E 58, 4737 (1998).

[9] T.P.C. van Noije and M.H. Ernst, Granular Matter 1, 57 (1998).

[10] T.P.C. van Noije, M.H. Ernst, E. Trizac, and I. Pagonabarraga, Phys. Rev. E 59, 4326 (1999).

[11] S.J. Moon, M.D. Shattuck, and J.B. Swift, Phys. Rev. E 64, 031303 (2001).

[12] P.K. Haff, J. Fluid Mech. 134, 401 (1983).

[13] J.R. Dorfman, T.R. Kirkpatrick, and J.V. Sengers, Annu. Rev. Phys. Chem. 45, 213 (1994).

[14] G. Grinstein, D.-H. Lee, and S. Sachdev, Phys. Rev. Lett. 64, 1927 (1990).

[15] A. Barrat, E. Trizac, and J.N. Fuchs, Eur. Phys. J. E 5, 161 (2001).

[16] S. Chapman and T.G. Cowling, The Mathematical Theory of Non-uniform Gases (Cambridge University Press, Cambridge, 1970).

[17] J.F. Lutsko, Phys. Rev. Lett. 86, 3344 (2001).

[18] R. Soto and M. Maréschal, Phys. Rev. E 63, 041303 (2001).

[19] B.J. Alder and T.E. Wainwright, Phys. Rev. Lett. 18, 988 (1967); Phys. Rev. A 1, 18 (1970); B.J. Alder and W.E. Alley,
Phys. Today 37, 56 (1984); E.G.D. Cohen, ibid. 37, 64 (1984); Am. J. Phys. 61, 524 (1993).

[20] J.R. Dorfman and H. van Beijeren, The Kinetic Theory of Gases, in Statistical Mechanics, Part B: Time-Dependent Processes, edited by B. J. Berne (Plenum Press, New York, 1977), Chap. 3.

[21] J.R. Dorfman and E.G.D. Cohen, Phys. Rev. Lett. 25, 1257 (1970).

[22] M.H. Ernst, E.H. Hauge, and J.M.J. van Leeuwen, Phys. Rev. Lett. 25, 1254 (1970).

[23] J.-P. Hansen and I.R. McDonald, Theory of Simple Liquids (Academic Press, London, 1986).

[24] S. McNamara and W.R. Young, Phys. Rev. E 50, R28 (1994); 53, 5089 (1996).

[25] E. Trizac and A. Barrat, Eur. Phys. J. E. 3, 291 (2000).

[26] M. Müller and H. J. Herrmann, in Physics of Dry Granular Media, edited by H. J. Herrmann, J.-P. Hovi, and S. Luding (Kluwer Academic Publishers, Dordrecht, 1998).

[27] S. Luding, Z. Angew. Math. Mech. 80, S9 (2000).

[28] I. Goldhirsch, M.L. Tan, and G. Zanetti, J. Sci. Comput. 8, 1 (1993).

[29] T.P.C. van Noije, M.H. Ernst, and R. Brito, Physica A 251, 266 (1998).

[30] M.P. Allen and D.J. Tildesley, Computer Simulation of Liquids (Clarendon Press, Oxford, 1987).

[31] L. Verlet and D. Levesque, Mol. Phys. 46, 969 (1982); N.F. Carnahan and K.E. Starling, J. Chem. Phys. 51, 635 (1969).

[32] J.J. Brey, J.W. Dufty, and A. Santos, J. Stat. Phys. 87, 1051 (1997).

[33] I.S. Gradshteyn and I.M. Ryzhik, Table of Integrals, Series, and Products (Academic Press, New York, 1965).

[34] J.M. Montanero and A. Santos, Granular Matter 2, 53 (2000).

[35] F. Rouyer, J. Martin, and D. Salin, Phys. Rev. Lett. 83, 1058 (1999).

[36] P. Deltour and J.-L. Barrat, J. Phys. I 7, 137 (1997).

[37] S. Luding and S. McNamara, Granular Matter 1, 113 (1998).

[38] I. Goldhirsch and G. Zanetti, Phys. Rev. Lett. 70, 1619 (1993).

[39] T.P.C. van Noije, M.H. Ernst, R. Brito, and J.A.G. Orza, Phys. Rev. Lett. 79, 411 (1997).

[40] M.L. Tan and I. Goldhirsch, Phys. Fluids 9, 856 (1997).

[41] S. Luding and H.J. Herrmann, Chaos 9, 673 (1999). 